Regina Rhodius | Monika Bachinger | Katharina Díaz Méndez | Susanne Ober

\title{
Transformative Lehre
}

Ein Leitfaden für den Einbezug von Praxisakteuren

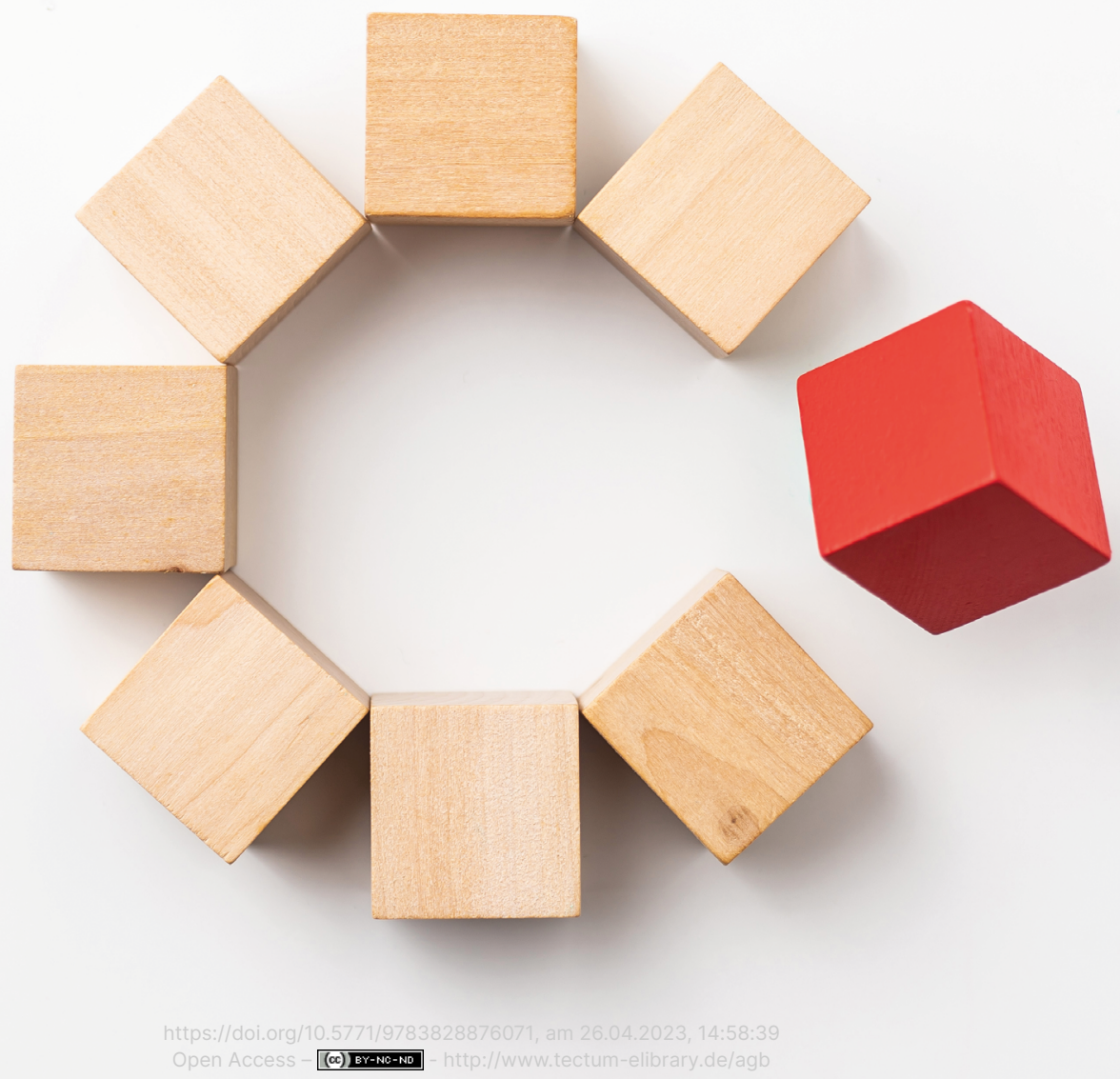


Rhodius | Bachinger | Díaz Méndez | Ober

Transformative Lehre 
Regina Rhodius | Monika Bachinger | Katharina Díaz Méndez | Susanne Ober

\section{Transformative Lehre}

Ein Leitfaden für den Einbezug von Praxisakteuren

Tectum Verlag 
Regina Rhodius | Monika Bachinger | Katharina Díaz Méndez | Susanne Ober Transformative Lehre

Ein Leitfaden für den Einbezug von Praxisakteuren

(c) Tectum Verlag - ein Verlag in der Nomos Verlagsgesellschaft, Baden-Baden 2022 ePDF 978-3-8288-7607-1

(Dieser Titel ist zugleich als gedrucktes Werk unter der ISBN 978-3-8288-4554-1 im Tectum Verlag erschienen.)

DOI: https://doi.org/10.5771/9783828876071

Umschlaggestaltung: Tectum Verlag, unter Verwendung

der Abbildung \# 1999737155 von domoko | www.shutterstock.com

Alle Rechte vorbehalten

Besuchen Sie uns im Internet

www.tectum-verlag.de

Bibliografische Informationen der Deutschen Nationalbibliothek

Die Deutsche Nationalbibliothek verzeichnet diese Publikation in der

Deutschen Nationalbibliografie; detaillierte bibliografische Angaben

sind im Internet über http://dnb.d-nb.de abrufbar.

\section{(c) (1) $\Theta \Theta$}

Dieses Werk ist lizenziert unter einer Creative Commons Namensnennung

- Nicht kommerziell - Keine Bearbeitungen 4.0 International Lizenz. 


\section{Inhalt}

Abbildungsverzeichnis VII

Tabellenverzeichnis VII

Danksagung IX IX

Gebrauchsanweisung XI

$\begin{array}{ll}\text { 1. Einführung in die transformative Lehre } & 1\end{array}$

2. Die Zusammenarbeit mit Praxisakteuren in der transformativen Lehre $\quad 7$

2.1 Das Reallabor Wissensdialog Nordschwarzwald als Erfahrungshintergrund $\quad 7$

2.2 Ziele und Mehrwerte der Zusammenarbeit mit Praxisakteuren 12

2.3 Die beteiligten Akteure 16

2.3.1 Lehrende in vielfältigen Rollen 17

2.3.2 Praxisakteure als Patinnen und Paten 18

$\begin{array}{ll}2.3 .3 \text { Studierende } & 20\end{array}$

3. Einbindung der transformativen Lehre an den Hochschulen 23

3.1 Einbindung der Lehre in ein Reallabor 23

3.2 Einbindung der Lehre in Studium und Curriculum 25 
Inhalt

4. Konkrete Bausteine für die Lehre 27

4.1 Einordnung der Bausteine in die Phasen eines Projektes 27

4.2 Die Steckbriefe 31

4.2.1 Phasenvorbereitende bzw. -unabhängige Bausteine 33

4.2.2 Co-Design-Bausteine 42

4.2.3 Co-Produktions-Bausteine 45

4.2.4 Co-Disseminations-Bausteine 49

4.2.5 Co-Evaluations-Bausteine 53

5. Und so könnte ein gesamtes Modul aussehen 57

$\begin{array}{ll}\text { Literaturverzeichnis } & 61\end{array}$

$\begin{array}{ll}\text { Die Autorinnen } & 67\end{array}$ 


\section{Abbildungsverzeichnis}

Abb. 1: Die Rollen transformativ Lehrender (eigene Darstellung)

Abb. 2: Die Rollen der Patinnen und Paten (eigene Darstellung)

Abb. 4: Der Aufbau der Steckbriefe (eigene Darstellung)

Abb. 5: Idealmodell, Variante 1 mit zwei zusammenhängenden Semestern

Abb. 6: Idealmodell, Variante 2 mit einer Dauer von insgesamt zwei Semestern

60

Der Baustein zu Beginn der Kapitel stammt aus der Abbildung \# 1999737155

von domoko | www.shutterstock.com

\section{Tabellenverzeichnis}

Tab. 1: Die Bausteine für die Zusammenarbeit mit Praxisakteuren

in der transformativen Lehre

Tab. 2: Zuordnung der 14 Bausteine zu den Transformationskompetenzen nach Wiek et al. (2011) 


\section{Danksagung}

Es war ganz toll zu sehen, wie viel Kreativität und Arbeit die Studierenden reinsteckten, und dass noch mal ganz eigene Ansätze zur Ursprungsidee vom 'Naturlabyrinth dazukamen. Da haben sie mit ihren Gedanken und Ideen das Projekt wirklich vorangebracht.

Martin Rimmler, Nationalpark Schwarzwald, WiNo-Projekt-Pate

Dieser Leitfaden basiert auf den Erfahrungen, die wir bei der Konzeption und Umsetzung von Lehrveranstaltungen innerhalb des Reallabors »Wissensdialog Nordschwarzwald « (WiNo) von 2016 bis 2020 sammeln konnten. Ausgehend vom Konzept des Forschenden Lernens entwickelten wir von Semester zu Semester unseren WiNo-Ansatz hin zu einer projektorientierten transformativen Lehre, in der die Zusammenarbeit mit Praxisakteuren vor Ort einen ganz besonderen Stellenwert hat.

Wir sind allen dankbar, die uns auf diesem Weg begleitet haben, uns wichtige Impulse und konstruktive Rückmeldungen gaben und unsere Begeisterung für dieses Format teilten:

- Frau Prof. Barbara Koch, Leiterin des Reallabors Wissensdialog Nordschwarzwald, die 2015 den Anstoß für die Einbindung von Lehrveranstaltungen in das WiNoProjekt an der Universität Freiburg gab und selbst ein Semester Lehre übernahm;

- den Studierenden an der Hochschule Rottenburg und der Universität Freiburg, die engagiert und beherzt alle Höhen und Tiefen einer selbstständigen Projektarbeit in Angriff nahmen; 
- den Patinnen und Paten Karin Beilharz, Dr. Susanne Berzborn, Sönke Birk, Dr. Ralph Bürk, Olfert Dorka, Alexander Feldberger, Diana Fritz, Viola Hajek, Thomas Hudeczek, Martin Rimmler, Dr. Dominik Rüede, Patrick Schreib, Axel Singer, Patrick Stader, Barbara Unruh und Antje Wurz, die den Studierenden mit zahlreichen Tipps und viel Engagement bei der Findung und Umsetzung von Projektideen zur Seite standen;

- Monika Krämer und Paul Zinser von der am Sachgebiet Tourismus des Landkreises Freudenstadt angesiedelten WiNo-Transfer-Stelle, die die Umsetzung von studentischen Projektergebnissen in die Wege leiteten;

- Hannes Rau für die Mitgestaltung der Lehre an der Hochschule Rottenburg in der ersten Förderperiode sowie Jakob Staneker, der die Lehre in Freiburg in der ersten Förderperiode organisatorisch unterstützte;

- Jonas Hinze und Dr. Reinhold John von der Forstlichen Versuchs- und Forschungsanstalt Baden-Württemberg, die ihre langjährige Expertise zum Thema Borkenkäfermanagement in die Lehre einbrachten;

- Antonietta Di Giulio und Rico Defila von der Forschungsgruppe Inter-/Transdisziplinarität an der Universität Basel für ihre wertvollen Rückmeldungen zu unserem ersten Leitfadenentwurf;

- Gabriele Haegele von der Hochschule Rottenburg sowie Silke Weiß und Rochelle Alsleben-Borrozino von der Hochschuldidaktik der Universität Freiburg für die fundierte Begleitung bei der Evaluation unseres Lehrformats.

- Dem Tectum Verlag sind wir dankbar für die professionelle Betreuung bei Korrektorat, Layout und Satz des Leitfadens. 


\section{Gebrauchsanweisung}

\author{
Es hat sehr viel Spaß gemacht, mit den Studierenden \\ zu arbeiten. Das war für mich natürlich auch einfach \\ etwas, das ich seit meiner Studienzeit vermisse, \\ sich an etwas festzubeißen, es wissenschaftlich \\ zu beleuchten und Hypothesen aufzustellen. \\ Und das fand ich total schön, \\ daran teilhaben zu dürfen. \\ Karin Beilharz, Praxis für Psychosoziale \\ Gesundheit, WiNo-Projekt-Patin
}

Unsere Studierenden, die sich in ihren jeweiligen Disziplinen ganz unterschiedlichen Herausforderungen bei der zukünftigen Gestaltung unserer Gesellschaft widmen, werden in ihrem späteren Berufsalltag selten mit Routineaufgaben konfrontiert sein. Ganz gleich, ob sie sich mit Fragen einer nachhaltigen Wirtschaftsweise, der Anwendung neuer Technologien, der Schaffung lebenswerter Siedlungs- und Erholungsräume oder der Organisation menschlichen Zusammenlebens befassen - es gilt, sehr komplexe Problemstellungen zu bewältigen. Wer sich aktiv in die anstehenden gesellschaftlichen Veränderungsprozesse (auch Transformationen genannt) einbringen will, muss bereit sein, sich mit anderen, ungewohnten Perspektiven auf ein Thema auseinanderzusetzen und mit Lösungen zu experimentieren. Für den Erwerb der nötigen Fertigkeiten und Kompetenzen braucht es daher bereits im Studium neben der Vermittlung von Grundlagenwissen ein experimentelles Setting, das wissenschaftliches Vorgehen, Praxisanbindung, Reflexion und gesellschaftliche Verantwortung miteinander verbindet - und damit eine transformative, auf Veränderung ausgerichtete Lehre ermög-
Gesellschaftliche Herausforderungen erfordern experimentelle Lehrsettings 
Der Aufbau des Leitfadens für transformative Lehre mit Praxisanbindung licht. Um diesem Anspruch gerecht zu werden, ist es aus unserer Sicht und Erfahrung zentral, Praxisakteure aus Wirtschaft, Verwaltung, Zivilgesellschaft und Politik in die Gestaltung einer transformativen Lehre zu integrieren. Transformative Lehre ist demnach in ihrem Kern transdisziplinär. Daher wird in dieser Publikation transdisziplinäre Lehre als obligatorischer Bestandteil transformativer Lehre verstanden.

Der vorliegende Leitfaden richtet sich an Dozierende verschiedener Hochschultypen, die transformative Lehrveranstaltungen gestalten und dabei insbesondere die Zusammenarbeit mit Praxisakteuren intensivieren wollen. Im Fokus des Leitfadens steht der im Rahmen des Reallabors Wissensdialog Nordschwarzwald entwickelte Ansatz der Zusammenarbeit mit Praxisakteuren im Rahmen von Patenschaften zwischen Praxisakteuren und Studierenden.

In Kap. 1 erläutern wir die Entstehungsgeschichte und die Ziele des Konzeptes der transformativen Lehre und diskutieren kritische Reflexionen des Konzeptes - ein Einstieg für alle, die prüfen wollen, ob sie dieses Konzept in ihrer Lehre einsetzen wollen.

Die Zusammenarbeit mit Praxisakteuren steht im Zentrum von Kap. 2. Anknüpfend an die Lehre im Reallabor des Wissensdialogs Nordschwarzwald als Ausgangspunkt des Leitfadens, werden Ziele und Mehrwerte einer solchen Zusammenarbeit sowie die beteiligten Akteure beschrieben - ein zentrales Kapitel, um die Voraussetzungen der Zusammenarbeit und die Rolle als Lehrende eingrenzen zu können.

Um transformative, praxisorientierte Lehre an Hochschulen umzusetzen, bedarf es gewisser Voraussetzungen. In Kap. 3 erläutern wir daher, welche strukturellen Rahmenbedingungen an den Hochschulen dafür hilfreich und welche Settings möglich sind - ein Kapitel, das Sie dabei unterstützen soll, den geeigneten Rahmen für Ihre Lehrveranstaltung zu finden, bzw. diese dem gegebenen Rahmen anzupassen. 
Wesentliche Bausteine eines Moduls bzw. Kurses ${ }^{1}$ sind in Kap. 4 steckbriefartig dargestellt und den unterschiedlichen Phasen eines transdisziplinären Projektes zugeordnet. Nach dem Baukastenprinzip können Sie sich hier auch nur einzelne Elemente für Ihren eigenen Kontext heraussuchen und individuell zusammenstellen.

Abschließend stellen wir in Kap. 5 zwei Möglichkeiten eines idealtypischen zweisemestriges Moduls vor. Dies soll Ihnen als Beispiel zur Veranschaulichung dienen, wie die einzelnen Bausteine ineinandergreifen könnten.

Wir wünschen Ihnen viel Erfolg und viel Freude bei der Zusammenarbeit mit Praxisakteuren in der transformativen Lehre. Über Feedback und Rückmeldungen zu Ihren Erfahrungen freuen wir uns. ${ }^{2}$

1 Den Begriff des Kurses verwenden wir für einsemestrige Veranstaltungen, den Begriff des Moduls für zweisemestrige, d.h. zwei Kurse umfassende Veranstaltungen.

2 Bitte senden Sie Ihr Feedback an: regina.rhodius@syntoslab.de. 


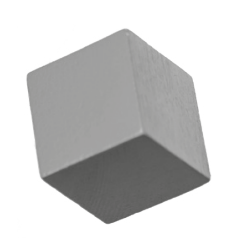

\section{Einführung in die transformative Lehre}

\author{
Da gab es einen regen Austausch, der beiden Seiten \\ ganz offensichtlich etwas gebracht hat und \\ bei dem sich dann auch Perspektiven \\ verändert haben - auf beiden Seiten. \\ Karin Beilharz, Praxis für Psychosoziale \\ Gesundheit, WiNo-Projekt-Patin
}

Bereits 2011 prägte der Wissenschaftliche Beirat Globale Umweltveränderungen (WBGU) den Begriff der »Großen Transformation «. Dieser Transformationsbegriff umfasst den gesamten "Wandel in Politik, Wirtschaft und Gesellschaft «, der notwendig ist, um »eine irreversible Schädigung der Weltökosysteme« durch das gegenwärtig praktizierte menschliche Wirtschaften zu vermeiden (WBGU 2011: 87). Bildung für die anstehende sozioökologische Transformation nimmt in dem entsprechenden Gutachten des WBGU eine zentrale Rolle ein. Sie soll transformativ sein, d.h. nicht nur dem Erwerb des für eine Transformation nötigen Wissens dienen, sondern selbst aktiv auf eine gesellschaftliche Transformation hinwirken und individuelle und kollektive Lernprozesse ermöglichen (WBGU 2011: 357ff.).

Auch auf internationaler Ebene spielt das Konzept der Transformation eine wichtige Rolle. Mit der Agenda 2030 verabschiedeten die Staats- und Regierungschefs der UN-Mitgliedsstaaten am 25. September 2015 ein ambitioniertes Programm für eine weltweite nachhaltige Entwicklung. Unter dem Motto »Transforming our world « wurden 17 Ziele für eine nachhaltige Entwicklung (Sustainable Development Goals, SDGs) formuliert, die auch den Bildungsbereich unter das Leitbild einer nachhaltigen Entwicklung stellen (UN 2015). Dieses Leitbild hat
Neue Ansprüche an die Hochschullehre durch die "Große Transformation" 
Transformative
Lehrformate
werden erprobt
Als Change Maker Interventionen im Nachhaltigkeitskontext durchführen bereits 2010 mit einer gemeinsamen Erklärung der deutschen Hochschulrektorenkonferenz und der Deutschen UNESCOKommission Eingang in den Hochschulbereich gefunden: Hochschulen sind aufgefordert, zur »zukunftsorientierten Entwicklung der Gesellschaft beizutragen « und »Bildung für nachhaltige Entwicklung zu einem konstitutiven Element in allen Bereichen ihrer Tätigkeit« zu machen (HRK und DUK 2010: 2).

Seit den Impulsen durch die Agenda 2030 und das WBGU-Gutachten hat sich auch in Deutschland eine rege Debatte über Bildung als ein Instrument der Transformation entwickelt, neue Bildungsformate wurden entwickelt und erprobt (GiBE und KommEnt 2018). Auch in der Fortbildung Lehrender ist transformative Lehre in den letzten Jahren bereits angekommen. ${ }^{3}$ An den Hochschulen sind oft auch studentische Initiativen aus dem Nachhaltigkeitskontext die Treiber neuer Lehr-Lernformate. ${ }^{4}$

Die Themen, die an verschiedenen Hochschulen mittels transformativer bzw. transdisziplinärer Lehransätze in den zurückliegenden Jahren adressiert wurden, sind vielfältig. Von Demokratieentwicklung, nachhaltiger Mobilitätskultur und Stadtgestaltung, Klimawandel, künstlicher Intelligenz bis hin zur Zukunft der Ernährung reicht das Spektrum der Themen. Die Erfahrungen mit dabei angewandten Lehrformaten wurden in Artikeln, Buchbeiträgen und Leitfäden festgehalten, reflektiert und für potenzielle Nachahmende aufbereitet (z. B. Albiez 2018, West 2018, Godat et al. 2018, Wanner et al. 2020).

Der Begriff der Intervention nimmt in diesen Beiträgen eine zentrale Rolle ein. Unter Interventionen verstehen wir jegliche Form von Impulsen, die bestehende Handlungsroutinen oder dominante Denkmuster hinterfragen und Alternativen einführen. Interventionen im Nachhaltigkeitskontext können ganz unterschiedlich aussehen:

3 Maßgebliche Impulse setzte z. B. das von 2010 bis 2020 aktive Bündnis für Hochschullehre Lehre ${ }^{n}$ [https://lehrehochn.de/].

4 Beispiele hierfür sind das netzwerk $n$ [https://www.netzwerk-n.org/201741/] sowie das an der Universität Freiburg angesiedelte Nachhaltigkeitsbüro [https://www.nachhaltigkeitsbuerofreiburg.de/. 
- Studierende hinterfragen in verschiedenen Themenfeldern wie Ernährung, Energie oder Konsum ihre eigenen Handlungsroutinen und verändern diese in Selbstexperimenten wie im Projekt »Klimaschutz gemeinsam wagen!« des Karlsruher Instituts für Technologie ${ }^{5}$.

- Studierende erfragen die Meinungen bestimmter Zielgruppen zu Nachhaltigkeitsthemen und reflektieren die Ergebnisse mit diesen gemeinsam - wie in unserem Projekt Wissensdialog Nordschwarzwald ${ }^{6}$.

- Studierende >zetteln $<$ Debatten durch Experimente im öffentlichen Raum `an $<$ - wie z. B. durch die Umwidmung von städtischen Parkplätzen in kleine Cafés und Bürgertreffs im Reallabor für nachhaltige Mobilitätskultur Stuttgart (RNM 2018) oder unter dem Motto »Wissen to Go« durch umgestaltete und in der Innenstadt positionierte Einkaufswagen (West 2018).

Durch die gezielte Anwendung von Interventionen sollen die Studierenden in den entsprechenden Bildungs- und Lehrformaten an Hochschulen befähigt werden, Kompetenzen für die Gestaltung von Transformationsprozessen zu erwerben. Als sogenannte Change Maker sollen sie zukünftig selbst Transformationsprozesse gestalten können (z. B. Krütli et al. 2018, Wanner et al. 2020). Häufig knüpft transformative Lehre dazu an Kompetenzmodelle aus dem Bereich der Bildung für nachhaltige Entwicklung (Bormann \& de Haan 2008) oder an Kompetenzmodelle der transdisziplinären Lehre an (Pearce et al. 2018). Seltener referenziert wird das Konzept der Gestaltungskompetenz nach de Haan (2008).

5 Einen Einblick in den Charakter der Selbstexperimente bietet die Website https://www.klimaschutzgemeinsamwagen.de/selbstexperimente/.

6 Ein Beispiel aus unserem Projekt Wissensdialog Nordschwarzwald findet sich unter https://www.schwarzwaelder-bote.de/inhalt.kreis-freudenstadtnationalpark-gedanke-noch-nicht-im-kollektiven-bewusstsein.61699el1f933-44cd-b5e0-cb8018647f07.html.

Vermittlung von Transformationskompetenzen als Ziel der Lehre 
Die Transformationskompetenzen nach Wiek et al. (2011) als Maßstab
Im Kompetenzmodell von Wiek et al. (2011) wurden die Kompetenzen zusammengeführt, die im Rahmen einer transformativen Lehre vermittelt werden sollen. Dieses speziell als Referenzrahmen für die Entwicklung von Lehrprogrammen, Evaluationen und Weiterbildungen im akademischen Bereich entwickelte Modell synthetisiert verschiedene Kompetenzmodelle aus dem Nachhaltigkeitskontext.

Kompetenz wird dabei definiert als ein »funktional verknüpfter Komplex aus Wissen, Fähigkeiten und Einstellungen, der eine erfolgreiche Aufgabenausführung und Problemlösung im Verhältnis zu realweltlichen Nachhaltigkeitsproblemen, -herausforderungen und -chancen ermöglicht« (Wiek et al. 2011: 204, eigene Übersetzung). Die Autorinnen und Autoren arbeiten, aufbauend auf einer breiten Literaturanalyse, die Kompetenzen heraus, die in der traditionellen Lehre weniger Berücksichtigung finden, aber für den Nachhaltigkeitskontext von besonderer Bedeutung sind. Ihr Kompetenzmodell umfasst die folgenden fünf Schlüsselkompetenzen (ebd.: 207ff., für Anwendung auf die Zusammenarbeit mit Praxisakteuren siehe Kap. 2.2):

- Systemisches Denken beinhaltet die Fähigkeit, komplexe Systeme zu analysieren und dabei unterschiedliche Systeme, Raumebenen sowie Dynamiken und Wechselwirkungen zwischen verschiedenen Komponenten einzubeziehen. Erforderlich ist hierfür eine profunde Kenntnis komplexer Systeme.

- Antizipative Kompetenz ermöglicht es, basierend auf Analyse und Evaluation, Zukunftsbilder zu entwerfen. Dafür bedarf es sowohl analytisch-kreativer Fähigkeiten als auch der Kenntnis spezifischer Tools, wie der Arbeit mit Szenarien. Im Hinblick auf Transformationsprozesse erlaubt Antizipation die Einschätzung der Wirkungen geplanter Interventionen.

- Normative Kompetenz umfasst die Fähigkeit, Werte, Prinzipien und Ziele zu diskutieren und zu reflektieren und bietet damit Orientierung für die Richtung der Transformation. Hierzu ist z. B. die Auseinandersetzung mit Fragen rechtlicher, ethischer und sozial-ökologischer Natur nötig. Dar- 
auf basierend lässt sich beurteilen, inwieweit ein Vorhaben tatsächlich zu mehr Nachhaltigkeit führt.

- Strategische Kompetenz dient der Planung und zielgerichteten Durchführung von transformativen Strategien, Interventionen und Maßnahmen. Sie beinhaltet die Erarbeitung von Plänen und Programmen und erfordert Kenntnisse im Bereich Projektmanagement und Organisationsentwicklung, z. B. auch in Bezug auf die Einbeziehung von Akteuren und den Umgang mit Barrieren.

- Interpersonale Kompetenz ist die Fähigkeit, Akteure zu motivieren und zu einer gemeinsamen Problemlösung zusammenzuführen. Hierzu sind ausgeprägte kommunikative Fähigkeiten, Verhandlungsfähigkeit und Führungsstärke ebenso nötig wie Empathie im Umgang mit Akteursgruppen aus ganz unterschiedlichen sozialen und kulturellen Herkünften.

Die Schlüsselkompetenzen greifen ineinander bzw. sind voneinander abhängig und bedürfen der Zusammenarbeit im Team. Probleme und Lösungsansätze sollen so ganzheitlich und rekursiv verstanden werden können. Die Ausrichtung der Lehre auf die oben erläuterten Kompetenzen stellt nicht nur spezielle Anforderungen an die Gestaltung der Lehrveranstaltungen. Sie erfordert auch adäquate Bewertungsmethoden, die z.B. auch reflexive Elemente beinhalten sollten (vgl. Richter 2006, Reis 2009 und Kap. 2.1).

Der Ansatz der transformativen Lehre wird in seinem normativen Anspruch im Sinne eines > Nachhaltigkeitsimperativs $<$ bei Fragen der Vermittlung von Nachhaltigkeit in der Lehre kritisiert.

Das Konzept der transformativen Lehre in der Kritik So wird beispielsweise befürchtet, dass der geradezu inflationäre Gebrauch des Begriffs zu dessen Aushöhlung führt (Grober 2013, Kropp 2019). Die Komplexität der verschiedenen Dimensionen von Nachhaltigkeit würden im Spannungsfeld zwischen theoretischer Vermittlung und praxisnahen Lösungen selten ausreichend reflektiert (Leal Filho 2018). Es gelte, darauf zu achten, dass Studierende nicht für Nachhaltigkeitsziele sindoktriniert sondern in die Lage versetzt werden, sich eine eigene Meinung zu bilden (BPB 2011). Auch würden wirtschafts- und wachstums- 
Konsequenzen der Kritik für transformativ Lehrende kritische Ansätze häufig ausgespart und Nachhaltigkeitsbildung sich zu sehr an Symptomen, nicht an Ursachen orientieren (Getzin \& Singer-Brodowski 2016). Getzin \& Singer-Brodowski (2016) regen daher die Unterscheidung in eine instrumentelle Bildung für nachhaltige Entwicklung und eine kritisch-emanzipatorische Bildung als nachhaltige Entwicklung an. Instrumentelle Bildung vermittele Wissen als Grundlage für Handlungsoptionen in Richtung einer sozial-ökologischen Transformation. Dagegen werden im kritisch-emanzipatorischen Ansatz Werte, Leitbilder und Normen von Transformation hinterfragt. Gefragt wäre eine »aktivierende, hinterfragende Lehrpraxis, die auf die Persönlichkeitsentwicklung der Studierenden fokussiert « (Thiele \& Becker 2020). Zudem kann der Ansatz, Studierende als von außen kommende Change Maker anzusehen, auf in die Lehre involvierte Praxisakteure auch befremdlich wirken.

Für unser Lehrkonzept beinhaltet die geschilderte Entstehungsgeschichte transformativer Lehre sowie deren kritische Reflexion zwei wesentliche Schlussfolgerungen. 1) Eine umfassende transformative Lehre muss sowohl eine intensive und kontroverse fachspezifische Debatte als auch die Auseinandersetzung mit Normen und Werten sowie ein hohes Maß an persönlicher Reflexion ermöglichen. 2) Eine solche Lehre erfordert von den Dozierenden eine offene und gegenüber dem Umfeld sensible Herangehensweise und die Kompetenz, gemeinsame Lernprozesse von Studierenden und anderen an der Lehre Beteiligten zu gestalten. 


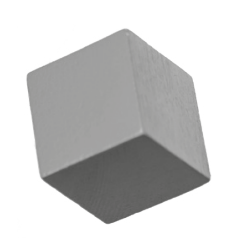

\title{
2. Die Zusammenarbeit mit Praxisakteuren in der transformativen Lehre
}

\author{
Das Interview mit dem Praxispartner hat zwar \\ all unsere Pläne zerhauen, aber trotzdem war es gut. \\ Anonymes Feedback einer/eines \\ Studierenden in der Evaluierung \\ Die Idee der Studierenden finde ich echt eine Supersache. \\ Insofern hat sich da die Sicht aufs Thema \\ bei uns schon geändert, dass man dann nochmal \\ andere kreative Möglichkeiten aufgezeigt bekommt, \\ jenseits dessen, was wir bislang so überlegt hatten. \\ Dr. Susanne Berzborn, Nationalpark \\ Schwarzwald, WiNo-Projekt-Patin
}

\subsection{Das Reallabor Wissensdialog Nordschwarz- wald als Erfahrungshintergrund}

Der vorliegende Leitfaden baut auf den Erfahrungen der Lehre im Reallabor Wissensdialog Nordschwarzwald (WiNo) auf. Ziel des vom baden-württembergischen Wissenschaftsministeriums von 2015 bis 2020 geförderten Reallabors war es, die Etablierung des am 1. Januar 2014 gegründeten Nationalparks Schwarzwald zu begleiten. Das Projekt verfolgte dabei zwei Blickrichtungen: Es fragte nach den Chancen, die der Nationalpark für die nachhaltige Entwicklung der ländlich geprägten Region bietet, und es wollte Schlüsse aus den im Nationalparkgebiet stattfindenden ökologischen Prozessen ziehen (Rhodius et al. 2020).

Lehre im Reallabor WiNo als Erfahrungshintergrund 
Ein transdisziplinäres Netzwerk als Basis der Lehre

Ziele der WiNo-Lehre

Themen und Projekte der WiNo-Lehre in Rottenburg
Als Reallabor geschah dies nicht in einem >klassischen Forschungsformat, sondern von Beginn an im Zusammenspiel zwischen Wissenschaft und Praxis. Demzufolge setzte sich das Team, das auch das Ausgangsnetzwerk für die ersten transdisziplinären Lehrveranstaltungen bildete, aus Wissenschafts- und Praxisinstitutionen zusammen. Unter der Leitung der Albert-Ludwigs-Universität Freiburg arbeiteten Forschende der Universität Freiburg, der Hochschule für Forstwirtschaft Rottenburg und der Forstlichen Versuchs- und Forschungsanstalt Baden-Württemberg zusammen. Als Praxispartner brachten sich die Verwaltungen des Nationalparks Schwarzwald und des Naturparks Schwarzwald Mitte/Nord, das Öko-Institut e. V., die EVOCO GmbH und das Landratsamt Freudenstadt sowie diverse Akteure der Nationalparkregion aus den Themenbereichen Tourismus und Regionalentwicklung ein.

Studierende der Hochschule Rottenburg und der Universität Freiburg wurden im Rahmen einer projektorientierten Lehre in die Reallaborarbeit integriert. Bereits in der ersten Förderphase (2015 bis 2017) zeigte sich, dass die kreativen Impulse der Studierenden in der Region auf eine hohe Resonanz stießen und die Studierenden gleichzeitig den Praxisbezug der Lehre schätzten. Aufgrund dieser Erfahrungen rückte das Format des Forschenden Lernens nach Schlicht (2013) mit Beginn der zweiten Förderperiode (2018 bis 2020) ins Zentrum der Arbeit des Wissensdialogs. Ziel dabei war es einerseits, Studierende zu befähigen, in konkreten und komplexen gesellschaftlichen Kontexten relevante Fragestellungen und Lösungsansätze in Bezug auf die nachhaltige Entwicklung ländlicher Regionen zu entwickeln und zu bearbeiten. Andererseits sollten die Erfahrungen in der Forschenden Lehre in die Entwicklung eines transformativen Lehrformates einfließen.

Insgesamt wurden zwölf einsemestrige Kurse an den beiden beteiligten Hochschulen konzipiert und durchgeführt, an denen insgesamt 220 Studierende teilnahmen. An der Hochschule Rottenburg fanden sechs Kurse zum Thema »Wald und Gesundheit « mit jeweils 2 SWS und 3 ECTS statt, die im Schwerpunkt »Tourismus« des Studiengangs Nachhaltiges Regionalmanagement verankert waren. Davon ausgehend, dass Wälder als Erholungsraum wertvolle Beiträge zur physischen und psy- 
chischen Gesundheit von Menschen leisten können, stand das Thema des »waldbasierten Gesundheitstourismus« im Mittelpunkt. Ziel der studentischen Projekte und Interventionen war es, gemeinsam mit regionalen Akteuren zu erkunden, wie waldbasierte Erholungsprozesse in attraktive touristische Angebote integriert werden können und wie gemeinsames Wissen zu einer waldbasierten touristischen Dienstleistungskette aufgebaut werden kann. So entwickelten die Studierenden auf Basis positiver Erholungswirkungen des Waldes exemplarische touristische Angebote, wie z. B. ein »Naturlabyrinth", einen »Atem-WaldErlebnispfad«, ein Angebot zum Thema »Wald als Ressource für das regenerative Stressmanagement « sowie das Erlebnisangebot »Ein Tag wilder «. Neben diesen Angeboten konnten in Kooperation mit der Gemeinde Bad Peterstal-Griesbach Vorschläge zu einem »Kur- und Heilwald« entwickelt werden.

An der Universität Freiburg wurden zwei Kurse im Bereich „Öffentlichkeitsarbeit und Wissenschaftskommunikation «, drei Kurse zum Thema »Nachhaltige Regionalentwicklung" sowie ein Kurs im Themenfeld »Borkenkäfer, Sturm und Dürre« mit jeweils 4 SWS und 5 ECTS angeboten.

Die Studierenden verschiedener Bachelorstudiengänge (z. B. Umweltnaturwissenschaften, Waldwirtschaft, Geographie) setzten sich zunächst mit der Frage auseinander, wie Wissenschaft besser in die und mit der Region kommunizieren kann, und entwickelten Kommunikationsinstrumente und Bildungskonzepte. Ab 2018 lag der Fokus im Bereich der nachhaltigen Regionalentwicklung, auf Vorschlag regionaler Akteure standen dabei auch digitale Ansätze zugunsten einer nachhaltigen Regionalentwicklung bzw. zum Erleben der Region im Blickpunkt. Die Studierenden setzten sich beispielsweise mit der Frage auseinander, wie der Gedanke der Nachhaltigkeit stärker in der Region verankert werden könnte. Sie entwickelten eine Wettbewerbsidee, bei der kommunale Projekte ausgezeichnet und publik gemacht werden, die Wege eines ressourcenschonenden Lebensstils aufzeigen. Teile dieser Idee fließen in das Projekt "Nachhaltige Kreisentwicklung« des Landkreises Freudenstadt ein. Unter dem Motto »Go Nature« konzipierte ein Studierendenteam eine Fotoplattform, die Digitalisierung, Naturerleben und regionale

Themen und

Projekte der

WiNo-Lehre

in Freiburg 
Das Patenmodell als zentrales Element der WiNo-Lehre

Weitere Elemente der WiNo-Lehre
Ökonomie miteinander verbindet. Für das Konzept »Altes neu erleben «, das anhand von Schautafeln auf Veränderungen in der Landschaft aufmerksam machen will, konnte bereits eine Gemeinde für die Umsetzung gewonnen werden, die in ein Angebot des Naturparks integriert wird. Zum Thema Müllvermeidung im Nationalpark wurden ein Barfußpfad konzipiert sowie ein Kurzfilm kreiert.

Die Gruppenarbeiten im Bereich »Borkenkäfermanagement« reichten von der Erstellung einer Gefährdungskarte für ausgewählte Waldbestände im Nordschwarzwald über die Erforschung des Einflusses von Borkenkäferbefall auf die touristische Entwicklung und die Auseinandersetzung mit zukünftigen waldbaulichen Konzepten zum Anbau der Fichte bis hin zu einer ersten Evaluierung des Borkenkäfermanagements im Umland des Nationalparks, dessen Ergebnisse von den Verantwortlichen bereits aufgegriffen wurden.

Für die Auswahl und Begleitung der studentischen Projektthemen konnten wir auf ein weitverzweigtes transdisziplinäres Netzwerk zurückgreifen, das im Rahmen der Reallaborarbeit entstanden war. Aus der ersten lockeren Mitarbeit von Praxisakteuren als Gastvortragenden oder Exkursionsführenden entwickelte sich im Projektverlauf das Patenmodell im Sinne der Einbindung von Praxisakteuren als Patinnen und Paten der Studierendenteams. Diese Zusammenarbeit mit Praxispatinnen und -paten stellte sich für unsere Lehre als zentral und besonders gewinnbringend heraus.

Darüber hinaus dienten Exkursionen in die WiNo-Region dazu, den Studierenden theoretische Inputs zu den Projektthemen an Praxisbeispielen begreifbar zu machen. Die Studierenden erhielten die Gelegenheit zum Peer-Feedback und wurden von den Lehrenden durch ein Projekt-Coaching intensiv begleitet. Die Bearbeitung der einzelnen Projektthemen fand außerhalb der Präsenzveranstaltungen statt. Die studentischen Gruppen waren angehalten, selbst einen Projekt- und Zeitplan für die Erarbeitung ihrer Aufgabenstellungen zu entwickeln. Die Vorstellung der Ergebnisse erfolgte jeweils am Ende des Semesters im Rahmen der Veranstaltungsreihe »Wissensdialog vor Ort« in der Region. 
Zur Bewertung der studentischen Leistungen wandten wir einen Bewertungsmix an, der den angestrebten Kompetenzerwerb widerspiegeln sollte (vgl. Kap. 1):

1. Die Studierenden reflektierten anhand von Leitfragen jeweils eine der Lehrveranstaltungen oder Exkursionen mit Praxispartnerinnen und -partnern; daraus erstellten sie ein gemeinsames Kurstagebuch.

2. Im Rahmen der Veranstaltungsreihe»Wissensdialog vor Ort» präsentierten die Studierenden ihre Gruppenergebnisse vor Praxisakteuren und interessierter Bürgerschaft und stellten sich der öffentlichen Diskussion.

3. Im Projektbericht stellten die Studierenden Projektverlauf und Ergebnisse schriftlich dar. Hier wurde neben einer schlüssigen Darlegung von Ziel- und Fragestellung sowie von Methodik und Ergebnissen auch Wert gelegt auf die Prüfung ihrer Ideen auf Umsetzbarkeit und auf eine offene Betrachtung des eigenen Lernprozesses während der Projektbearbeitung.

Um die gewonnenen Erfahrungen für die Entwicklung eines didaktischen Konzeptes fruchtbar machen zu können, wurden an den beiden beteiligten Hochschulen verschiedene Aktivitäten zur Evaluation und Dokumentation der Lehre im Wissensdialog Nordschwarzwald durchgeführt. Zum Zeitpunkt der Evaluierung arbeiteten wir noch nicht mit dem Kompetenzmodell nach Wiek at al. (2011), sondern bezogen uns, basierend auf einer Literaturanalyse, auf die folgenden Kompetenzbereiche für die quantitative Erhebung der Selbsteinschätzung des Kompetenzzuwachses der Studierenden: Fachkompetenz, Methodenkompetenz, Präsentationskompetenz, Kommunikationskompetenz und Kooperationskompetenz. Diese Bereiche weisen Überschneidungen mit dem Kompetenzmodell nach Wiek et al. (2011) auf. So wurden z. B. Verhandlungsfähigkeiten oder kommunikative Fähigkeiten, wie Wiek et al. sie unter »interpersonaler Kompetenz « zusammenfassen, in unserem Fragebogen unter den Bereichen »Kooperationskompetenz « und »Kommunikationskompetenz« abgefragt.
Bewertung der studentischen Leistungen

Systematische Evaluierung der Lehre 
Fragebogen, Reflexionsgespräche und -workshop als

Evaluierungsmethoden

Zusätzlich zu der quantitativen Datenerhebung wurden qualitative Feedbacks der Studierenden erhoben. Dies erfolgte in ca. zweistündigen Reflexionsworkshops mit den Studierenden jeweils am Ende der Lehrveranstaltungen. Die in die Lehre involvierten Patinnen und Paten aus der Praxis brachten ihr Feedback und ihre Empfehlungen für die weitere Entwicklung des Lehrkonzepts in qualitativen halbstandardisierten Interviews, den Reflexionsgesprächen, zum Ausdruck. Unsere Erfahrungen als Lehrende wurden in einem eintägigen Reflexionsworkshop gebündelt und systematisch aufgearbeitet. Auf diesem Fundament entwickelten wir Lehrende das hier vorliegende Lehrkonzept und diskutierten dieses mit den Patinnen und Paten sowie interessierten Praxispartnern.

\subsection{Ziele und Mehrwerte der Zusammen- arbeit mit Praxisakteuren}

Transformativer Kompetenzerwerb im Patenmodell

Grundzüge der Zusammenarbeit im Patenmodell
In transformativen Projekten ist es Ziel, die Erarbeitung von Problemlösungen für gesellschaftliche Herausforderungen und die Gewinnung wissenschaftlicher Einsichten zur Nachhaltigkeitstransformation miteinander zu verbinden (»to integrate the pathway of problem solution ‘ and the 'pathway of scientific innovation « ; Lang et al. 2012). Auch transformativ ausgerichtete Lehrprojekte erarbeiten praxisrelevante Problemlösungen. Sie dienen jedoch nicht dem generellen wissenschaftlichen Erkenntnisgewinn, sondern dem Kompetenzerwerb der Studierenden. Im Folgenden sollen daher die Ziele und Mehrwerte, die die Zusammenarbeit mit Patinnen und Paten für den Kompetenzerwerb der Studierenden ermöglichen, skizziert werden. So können Dozierende, die daran interessiert sind, transformative Lehrkonzepte zu entwickeln und durchzuführen, sich einen Überblick verschaffen, ob das Patenmodell für ihre individuellen Lehrziele geeignet ist.

Spezifisches Ziel des Patenmodells ist es, Akteure aus Wirtschaft, Verwaltung, Zivilgesellschaft und Politik in die Gestaltung der Lehre zu integrieren und den Studierenden damit eine ausgeprägte Praxisorientierung zu ermöglichen. Die Praxisakteure sind dabei keine einmaligen Gäste einzelner Lehrveranstaltungen, 
sondern das gesamte Semester hinweg als Patinnen und Paten von Studierendengruppen in die Lehre eingebunden. Dem Patenmodell zugrunde liegt ein projektorientierter Lehransatz, in dem Studierende in Kleingruppen eigenständig ihre Projektthemen bearbeiten. Die Problem- und Fragestellungen ihrer Themen gehen entweder auf Vorschläge aus der Praxis oder von Lehrkräften zurück oder werden von den Studierenden zu Beginn des Semesters nach der inhaltlichen Einführung selbst erarbeitet. Jede Studierendengruppe wird während des gesamten Arbeitsprozesses nicht nur von der Lehrkraft, sondern von einem Praxisakteur oder einer Praxisakteurin begleitet, der oder die damit als Pate oder Patin der Gruppe fungiert.

Studierende sollen fachliche und methodische Kompetenzen gewinnen, diese praktisch anwenden und - in Bezug auf den transformativen Charakter der Lehre - lernen, , Veränderungsprozesse $<$ zu gestalten. Die in Kapitel 1 vorgestellten Transformationskompetenzen nach Wiek et al. (2011) finden sich beispielhaft wie folgt wieder:

- Systemisches Denken: Studierende lernen komplexe Problemstellungen durch die Augen der Patinnen und Paten kennen. Sie erfahren von Wechselwirkungen zwischen verschiedenen Bereichen wie Naturschutz, Regionalentwicklung und Tourismus. Über die Netzwerke der Patinnen und Paten können sich die Studierenden zudem mit Meinungen und Einstellungen verschiedener Stakeholder vertraut machen und gewinnen dadurch Einblicke in die Komplexität sozialer Systeme. Insgesamt werden sie so befähigt, in konkreten und komplexen gesellschaftlichen Kontexten relevante Fragestellungen in Bezug auf das Thema nachhaltige Entwicklung zu identifizieren.

- Antizipative Kompetenz: Gemeinsam mit den Patinnen und Paten suchen die Studierenden nach zukunftsfähigen Lösungen und erlernen das Denken in Szenarien. Dabei lernen sie von den Patinnen und Paten auch, die Machbarkeit von Projektinhalten in den Blick zu nehmen.

- Normative Kompetenz: In der Auseinandersetzung mit den Patinnen und Paten erfahren sie, welche Normen und Werte
Vermittlung von Schlüsselkompetenzen im Patenmodell 
Höhere Motivation der Studierenden und größere Offenheit der Praxisakteure für diese handlungsleitend sind. Nicht immer sind diese Normen und Werte mit dem Ziel einer nachhaltigen Entwicklung in Einklang zu bringen. Dieses Spannungsfeld auszuhalten und im Wissen um Werte und Normen nach kreativen Lösungen zu suchen, sind weitere Lernziele der Zusammenarbeit zwischen den Studierenden und den Patinnen und Paten.

- Strategische Kompetenz: Die Projektbearbeitung führt die Studierenden an Grundlagen des Projekt- und Zeitmanagements heran. Sie lernen zudem, wissenschaftliche Arbeitstechniken und Kenntnisse auf reale Problemstellungen anzuwenden. Gleichzeitig werden sie mit den Erfahrungen und Anregungen ihrer Patin oder ihres Paten konfrontiert und lernen, sich dazu zu positionieren.

- Interpersonale Kompetenz: Als wesentlicher Mehrwert gilt der Erwerb von sozialen Kompetenzen wie Kommunikationsfähigkeiten gegenüber dem Team und der Patin oder dem Paten sowie die Sicherheit im Umgang mit Öffentlichkeit und im Vortrag von Ergebnissen. In der gemeinsamen Projektplanung mit den Patinnen und Paten ist zudem Verhandlungsgeschick gefragt.

Die Ergebnisse der Studierenden landen nicht >in der Schublade $<$, sondern werden von den Patinnen und Paten weiterverwandt. Dieses Wissen und das von Patinnen und Paten begleitete Arbeiten ermöglichen erfahrungsgemäß auch eine ungewöhnlich hohe Identifikation der Studierenden mit dem Projekt und damit Motivation und Eigenaktivität. Dadurch entstehen oft erstaunlich detaillierte Beiträge zu den Fragestellungen, die bei den Praxisakteuren Anerkennung finden. Zugleich ist die Offenheit von Praxisakteuren und Bürgerschaft gegenüber Studierenden höher als gegenüber Expertinnen und Experten. Dadurch ist die Zusammenarbeit mit Studierenden ausgesprochen geeignet, öffentliche Diskurse auch zu sonst konfliktbehafteten Themen anzuregen.

Im Ergebnis der Zusammenarbeit von Studierenden, Lehrenden und Patinnen bzw. Paten sollen konkrete, innovative Lösungsansätze für (regionale) Problemstellungen entstehen, die der Pra- 
xis zugutekommen. Bis zu einem gewissen Grad kommen die studentischen Projekte diesem Wunsch auch nach: Die Patinnen und Paten erhalten kreative Inputs der Studierenden und frische Perspektiven auf bestehende Herausforderungen. Patinnen und Paten werden vor diesem Hintergrund angeregt, die eigenen Einstellungen oder Sichtweisen zu verändern.

Dies trifft ebenso auf die Lehrenden zu, die sich in ihrer Lehre durch den Anspruch der Praxistauglichkeit des vermittelten Wissens stärker hinterfragt sehen. Sie erhalten durch den Austausch mit den Patinnen und Paten Zugang zu Praxiswissen, können im Rahmen ihrer Lehre an Nachhaltigkeitslösungen mitwirken und sich ein eigenes Netzwerk regionaler Akteure aufbauen. Im Austausch mit Studierenden, Patinnen und Paten haben sie die Chance, ein weniger akademisch ausgeprägtes, sozial robustes Transformationsverständnis zu entwickeln.

Transformatives Lernen findet damit nicht nur in Bezug auf gesellschaftliche Veränderungen statt, sondern betrifft gleichermaßen Lernprozesse und Eigenwahrnehmungen von Studierenden, Lehrenden und Dozierenden.

Wenn die Lehre in ein Forschungsprojekt eingebunden ist, kann daraus auch der Anspruch erwachsen, dass die Lehre nicht nur den geschilderten praxisorientierten und didaktischen Zielen, sondern zudem der Generierung wissenschaftlicher Erkenntnisse für das Forschungsprojekt dienen soll. Unserer Erfahrung nach kann dies leicht zu einer Überforderung der Studierenden und einer Überfrachtung des an sich bereits anspruchsvollen Lehrformats führen. Daher empfehlen wir die Konzentration auf die Kerndimensionen »Kompetenzerwerb der Studierenden ermöglichen « und »Praxislösungen erarbeiten «. Die Ableitung von wissenschaftlichem Erkenntnisgewinn aus den studentischen Projekten sollte begleitend durch wissenschaftliche Projektmitarbeitende erfolgen.

Denn bereits die beiden Kernziele erzeugen ein Spannungsfeld, das sich an verschiedenen Stellen des Lehrformats zeigt. So stellen Patinnen und Paten Ansprüche an die Umsetzungsreife studentischer Arbeiten, die diese vor dem Hintergrund der erst noch zu
Konzentration auf zwei Zieldimensionen

Spannungsfelder zwischen Praxisorientierung und Kompetenzerwerb 
erwerbenden Kompetenzen unter Umständen nicht leisten können (vgl. Hilger \& Keil 2021). Studierende müssen Ansprüche an die Projektbearbeitung sowohl aus wissenschaftlicher als auch aus praktischer Sicht erfüllen. Sie sollten dabei die Möglichkeit haben, Fehler zu machen. Es ist daher wichtig, gegenüber Patinnen und Paten zu kommunizieren, dass die studentischen Projektarbeiten einen Lern- und Experimentiercharakter aufweisen und die Studierenden in diesen Lehrformaten in erster Linie Lernende und keine Dienstleister sind. Zugleich sind Studierende aber auch gefordert, im Umgang mit den Patinnen und Paten Professionalität zu entwickeln.

Lehrende müssen die Rahmenbedingungen und Limitationen von Patinnen und Paten ebenso in ihrer Projektplanung berücksichtigen wie die Anforderungen an eine gute Didaktik. Sie sollten klar kommunizieren, welche Herausforderungen transformative Lehrprojekte beinhalten, welche Mehrwerte für die beteiligten Akteure entstehen können und welche Aufgaben auf die Akteure zukommen. All diese Abstimmungs- und Kommunikationsprozesse nehmen viel Zeit in Anspruch, so dass der Zeitaufwand im Vergleich zur üblichen Vor- und Nachbereitungszeit von Lehrveranstaltungen deutlich höher ist.

\subsection{Die beteiligten Akteure}

Frühzeitige Abklärung von Erwartungen und Aufgaben und deren kontinuierliche Kommunikation notwendig
Lehrende, Patinnen bzw. Paten und Studierende sind die drei zentralen Akteursgruppen der transformativen Lehre mit dem Patenmodell. Lehrende und Patinnen bzw. Paten geben den Rahmen der studentischen Projektarbeiten vor, der von den Studierenden ausgefüllt, genutzt, ggf. auch verändert wird. An alle drei Akteursgruppen werden in der Zusammenarbeit Erwartungen und Aufgaben herangetragen, die für die Beteiligten teils neu und ungewohnt sind. Daher ist es zentral, zu Beginn einer Lehrveranstaltung ausreichend Zeit in das Abklären gegenseitiger Erwartungen und Aufgaben sowie die Definition der spezifischen Rollen zu investieren - eine Aufgabe, die den Lehrenden zukommt (siehe Baustein 1). Erfolgt dieser Schritt nicht, können Lehrende, Studierende und Praxispartner mit ganz unterschied- 
lichen Erwartungen an die studentischen Projekte herangehen und so Missverständnisse entstehen, die die Zusammenarbeit erschweren. Die getroffenen Absprachen sollten für alle transparent festgehalten werden, z. B. in Form von Akteurssteckbriefen und Meilensteinplänen (siehe Kap. 4, Baustein 1).

\subsubsection{Lehrende in vielfältigen Rollen}

Bereits in «klassischen « projektorientierten Seminaren sind Lehrende nicht nur für die Wissensvermittlung, sondern auch für die Zusätzliche Rollen der Lehrenden Gestaltung und Begleitung von Lern- und Arbeitsprozessen verantwortlich. In der transformativ-transdisziplinären Lehre nimmt die Verantwortung für die Prozessgestaltung weiter zu. Dies liegt zum einen im Anspruch begründet, dass transformative studentische Projekte in der Praxis Wirkung entfalten und zugleich auch wissenschaftlichen Standards genügen sollen. Aufgabe der Lehrenden ist es, die Studierenden bei der Bewältigung dieses Spagats und einer realistischen Projektplanung zu unterstützen und kontinuierlich zu begleiten. Zum anderen kommen mit den Patinnen und Paten neue Akteure ins Spiel, der Abstimmungsbedarf steigt. So gilt es z. B. Termine zu vereinbaren, Zwischenergebnisse zu diskutieren oder bei auftretenden Unstimmigkeiten zwischen Studierenden und Patinnen bzw. Paten zu vermitteln.

Die Rollen, die den Lehrenden aus diesen Aufgaben erwachsen, sind damit vielfältig (siehe Abb. 1): Sie sind Prozessmotoren und `Regisseure $\triangleleft$ des Gesamtprozesses, zugleich müssen sie die Qualität des zu vermittelnden Wissens und Möglichkeiten des Kompetenzerwerbs garantieren (siehe auch Müller-Christ et al. 2018). In der Begleitung der Studierenden agieren sie als Coaches; in der Zusammenarbeit mit Patinnen und Paten sind sie Koordinierende und Netzwerkende. Als Moderierende leiten sie Gruppenprozesse an und formulieren Spielregeln und Strategien, wie mit Konflikten, die durch unterschiedliche Interessenlagen entstehen können, umgegangen wird. Wenn nötig, moderieren sie den Austausch zwischen Studierenden und Patinnen bzw. Paten oder betätigen sich gar als Konfliktmanagerinnen und -manager. Teilweise kollidieren diese Rollen mit der klassischen Bewertungsaufgabe von Lehrenden. 
Vielfalt an

Qualifikationen der Lehrenden erforderlich

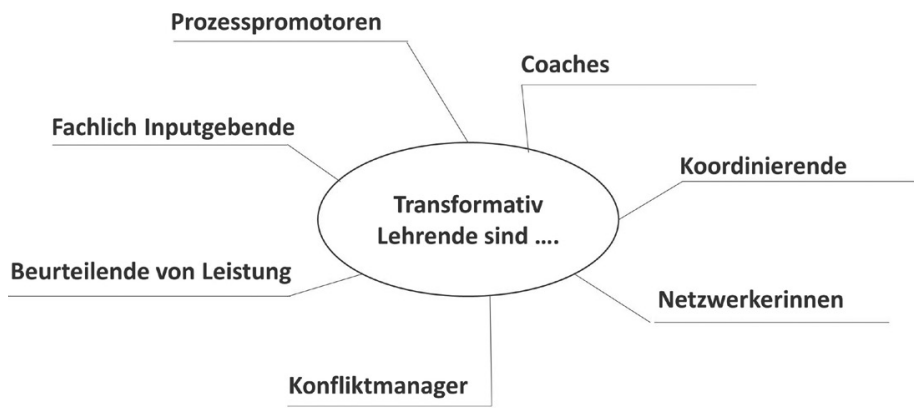

Abb. 1: Die Rollen transformativ Lehrender (eigene Darstellung)

Um der Vielfalt dieser verschiedenen Rollen gerecht werden zu können, brauchen Lehrende zahlreiche Qualifikationen. Sie benötigen die Fähigkeit, verschiedene Vorstellungen und Interessen bei der Planung und Durchführung des Projektes zu berücksichtigen, sie zu vereinbaren und tragfähige Kompromisse zu erreichen. Sie benötigen zudem fachliche und methodische Expertise, um die erforderliche inhaltliche Einbindung der Projekte leisten und Studierende und Patinnen bzw. Paten bei der Durchführung ihrer Projekte unterstützen zu können. Dabei ist nicht immer alles plan- und steuerbar. Lehrende sind gefordert, Unerwartetes zuzulassen und Änderungen mit einem hohen Flexibilitäts- und Reflexionsgrad anzunehmen. Nicht zuletzt bedeutet dies auch die Offenheit, eigene Standpunkte aufzugeben.

\subsubsection{Praxisakteure als Patinnen und Paten}

Praxisperspektive für Studierende

Bei der Erkundung von Problemstellungen benötigen Studierende nicht nur die Begleitung durch Lehrende, sondern auch die Prüfung ihrer Ideen auf Praxistauglichkeit, die am besten durch Praxisakteure erfolgen kann. Diese stehen jeweils einem Studierendenteam als Patinnen und Paten zur Verfügung und können als Fachpromotoren verstanden werden. Als zusätzliche Betreuende bzw. Ansprechpersonen der Studierenden unterstützen sie das Vorankommen des studentischen Projektes mit Rat und Tat. Patinnen und Paten sind Fachleute für konkrete (regionale) Fragen. Sie verfügen über fachliche Expertise und Praxiserfahrungen, aber auch über intensive Regionalkenntnis. 
Sie steuern zudem Kontakte zu weiteren fachlichen oder regionalen Inputgebenden bei.

Konkret interagieren Patinnen und Paten sowie Studierende über das gesamte transdisziplinäre Lehrprojekt hinweg. Die Patinnen und Paten bringen Fragen aus ihrer Praxis als Aufgaben für die Studierendengruppen ein. Dabei wählen sie in der Regel Fragen aus, zu denen sie ganz bewusst Impulse junger Menschen suchen. Sie konkretisieren gemeinsam mit Lehrenden und Studierenden das zu bearbeitende praxisrelevante Problem.

Auch auf die Patinnen und Paten kommen damit ganz verschiedene Rollen zu (siehe Abb. 2): Sie sind Impulsgebende und Fachleute, Coaches und Motivierende, Netzwerkende und Türöffner in die Region. Anders als Lehrende übernehmen sie die Patenrolle meist nicht als Teil ihrer regulären Tätigkeit, sondern als zusätzliche Aufgabe, deren Umfang daher auch überschaubar bleiben sollte.

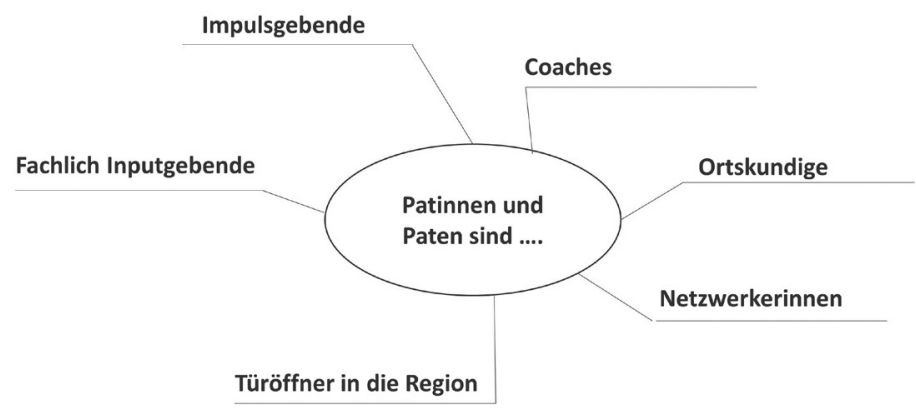

Abb. 2: Die Rollen der Patinnen und Paten (eigene Darstellung)

Patinnen und Paten benötigen eine große Offenheit und Flexibilität, da Studierende die Freiheit haben, die ihnen gestellte Aufgabe eigenständig zu interpretieren und zu lösen, und nicht als Auftragnehmende fungieren.

Der Erfolg der studentischen Projekte stellt sich vor allem dort ein, wo sich Patinnen und Paten als Mentorinnen und Mentoren der Studierenden verstehen und Freude an der Begleitung junger Menschen haben. 
Studierende im Spannungsfeld zahlreicher Erwartungen

\subsubsection{Studierende}

Studierende sind die zentralen Akteure in transformativen Lehrprojekten. Die Ergebnisse der Projekte und ihre Impulskraft für die Praxis hängen vom Engagement sowie der Qualität der Inputs der Studierenden ab. Sie sind zugleich Lernende, aber auch Gestaltende und Wissen Generierende (siehe Abb. 3). Aufgrund der Verortung der Lehrprojekte an der Nahtstelle zwischen Lehre und Praxis finden sich die Studierenden dem Spannungsfeld gegensätzlicher Erwartungen ausgesetzt. Zum einen sollen sie dem Anspruch gerecht werden, forschend und empirisch arbeitend Antworten auf die Fragestellungen ihrer Projekte zu finden. Zum anderen sehen sie sich den Ansprüchen der Patinnen und Paten gegenüber, möglichst sichtbare Ergebnisse oder Veränderungen zu generieren und sich bereits weitgehend professionell im Praxisfeld zu bewegen. Nicht zuletzt geht es für die Studierenden darum, formulierte Kompetenz- und Prüfungsziele zu erreichen.

Im Projektverlauf durchlaufen Studierende unterschiedliche Arbeitsphasen: Nach der Auswahl ihres Themas erarbeiten sie einen konkreten Arbeits- und Zeitplan, wählen geeignete Methoden zur Beantwortung ihrer Fragestellungen aus, wenden kreative Problemlösungstechniken an, tragen Ergebnisse zusammen, interpretieren und präsentieren diese. Arbeits- und Lernprozesse verlaufen dabei nicht immer linear, sondern in reflexiven Schleifen. Zu diesen typischen Arbeitsschritten einer projektorientierten Arbeit kommen Abstimmungsprozesse mit der Patin oder dem Paten hinzu. Der Anspruch, mit der eigenen Arbeit einen konkreten Beitrag zur Nachhaltigkeitstransformation zu leisten, führt zu einem häufigen Hinterfragen von Projektausrichtung und -stand.

Studierende vor Überforderung schützen
Insgesamt erfordert die Projektbearbeitung von den Studierenden ein hohes Maß an Selbstständigkeit und Autonomie, Frustrationstoleranz, Team- und Koordinierungsfähigkeit, methodische und fachliche Kompetenzen sowie Ergebnisorientierung. Die Bearbeitung dieser Art von Projekten bedeutet in der Regel im Vergleich zu klassischen Seminararbeiten ohne Praxisorientierung mehr Arbeitsaufwand für die Studierenden, auch 
im Hinblick auf die Kommunikation mit den Patinnen und Paten. Angesichts dieser vielfältigen Ansprüche sollten Lehrende Studierende auch vor überhöhten Anforderungen schützen und deutlich machen, dass im Vordergrund der eigene Lernprozess steht und trotz der Außenwirkung der Projektergebnisse auch Fehler erlaubt sind. Auch könnten die Lehrenden neben den erwähnten Vorgaben zur Häufigkeit der Kontaktaufnahme zwischen Patinnen bzw. Paten und Studierenden dafür Sorge tragen, dass innerhalb der Studierendengruppe eine Projektkoordinatorin bzw. ein Projektkoordinator benannt wird, die bzw. der die Kommunikation mit der Patin oder dem Paten organisiert. In jedem Fall sollte seitens der Lehrenden nicht nur gegenüber den Patinnen und Paten ein klares Erwartungsmanagement erfolgen, sondern auch in der Zusammenarbeit mit den Studierenden.

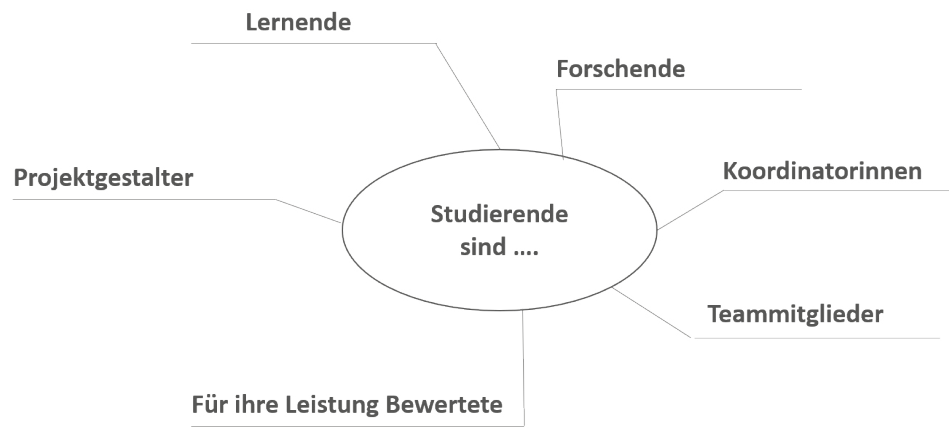

Abb. 3: Die Rollen der Studierenden (eigene Darstellung) 


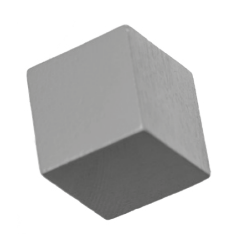

\section{Einbindung der transformativen Lehre an den Hochschulen}

Ich wünsche mir für mein Studium mehr Projekte, bei denen die Ergebnisse nicht einfach in der Schublade landen.

Anonymes Feedback einer/eines

Studierenden in der Evaluierung

Den Gesprächen mit den Studierenden war zu entnehmen, dass diese je nach Studiengang und Studienfortschritt in eine

Vielzahl von verschiedenen Projekten eingebunden sind.

Dadurch war die zeitliche Abstimmung auch innerhalb der Gruppen nicht immer einfach. Für den begrenzten Zeithorizont konnten aber insgesamt doch recht gute Ergebnisse erzielt werden.

Thomas Hudeczek, Gemeinde Forbach, WiNo-Projekt-Pate

In welchem Maß die Ziele transformativer Lehre erreicht werBedeutung den können, ist stark von den Rahmenbedingungen abhängig, in die entsprechende Lehrveranstaltungen an den Hochschulen der Rahmenbedingungen und Studiengängen eingebettet sind. Dabei können strukturelle Rahmenbedingungen von organisatorischen und ressourcenbezogenen Rahmenbedingungen unterschieden werden.

\subsection{Einbindung der Lehre in ein Reallabor}

Unter das Thema der strukturellen Rahmenbedingungen fällt insbesondere die Frage, ob und inwieweit transformative Lehre in übergeordnete Lehr- oder Forschungskontexte eingebunden werden kann. Als besonders vorteilhaft für transformative Lehre

Reallabore als attraktiver struktureller Rahmen 
erweist sich die Einbettung in ein Reallabor-Setting, das organisatorisch und ressourcenbezogen viele Vorteile mit sich bringt.

Der Begriff Reallabor macht deutlich, dass die Forschung nicht fernab der Alltagswelt der Menschen, sondern mitten in deren realem Leben stattfindet. Zugleich wird eine Brücke zu der naturwissenschaftlichen Arbeit im Labor geschlagen: Dieser ähnlich, soll in einem Reallabor systematisch experimentiert werden nicht mit chemischen Stoffen oder physikalischen Ansätzen, sondern mit Verhaltensweisen, neuen Denkansätzen, unkonventionellen Problemlösungen. Die im Kontext alltäglichen Lebens angesiedelte experimentelle Arbeitsweise soll sicherstellen, dass gesellschaftliche Veränderungen nicht nur untersucht, sondern innerhalb des Reallabors selbst initiiert, begleitet und reflektiert werden (Schneidewind \& Singer-Borowski 2014). In Reallaboren werden damit Forschungs-, Praxis- und Bildungsziele gleichermaßen verfolgt (Beecroft et al. 2018, Beecroft 2020): Es gilt, Wissen über die Transformation zu generieren (Forschungsziele), konkrete Projekte und Prozesse der Transformation anzustoßen (Praxisziele) und individuelle und kollektive Lernprozesse zu ermöglichen (Bildungsziele). Wie bereits geschildert, konzentriert sich transformative Lehre auf Praxis- und Bildungsziele, eigene Beiträge zur Forschung werden nicht angestrebt.

Weites Reallabore sind dabei nicht per se auf das Thema der nachthematisches spektrum möglich haltigen Entwicklung festgelegt, sondern können auch andere gesellschaftlich legitimierte, ethisch gut begründete und gemeinwohlorientierte Ziele, wie z. B. Integrationspolitik oder technologische Entwicklungen, in den Blick nehmen (Defila \& Di Giulio 2018). Dies öffnet auch das thematische Spektrum der möglichen Einbindung von Reallaboren in die Lehre.

Reallabore als Katalysatoren studentischer Projekte
Mit ihrem Anspruch, gesellschaftliche Herausforderungen mithilfe eines experimentellen Forschungsdesigns zu bearbeiten, eng mit Praxisakteuren zu kooperieren und Raum für Reflexion zu ermöglichen, bieten Reallabore einen attraktiven Rahmen für die Lehre. Studierende finden sich so thematisch in einem größeren Kontext wieder und können in ihren eigenen Projekten auf Ansprechpersonen, bereits erhobene Daten oder Forschungsinfrastruktur zurückgreifen. Dadurch können die studentischen 
Projekte einen schnellen Take-off - also einen schnellen Start verwirklichen. Zudem hilft die Einbettung den Studierenden, den Wert der eigenen Beiträge für größere gesellschaftliche Fragestellungen zu verstehen. Das Sense Making, also das Verstehen von größeren Zusammenhängen und die Einordnung der eigenen Beiträge zur Lösung gesellschaftlicher Probleme, fällt dadurch besonders leicht. Teil eines größeren Forschungsrahmens zu sein, erlaubt es den Lehrprojekten, von organisatorischen Strukturen und Kontakten zu profitieren. Kosten, die den Patinnen und Paten sowie den Studierendengruppen entstehen (z.B. Fahrt- und Übernachtungskosten) können durch Mittel des Reallaborprojektes beglichen werden.

\subsection{Einbindung der Lehre in Studium und Curriculum}

Auch die Verankerung von transformativer Lehre im Studienverlauf der Studierenden stellt besondere Anforderungen. Grundsätzlich profitieren transformative Lehrprojekte von einer hohen Interdisziplinarität innerhalb der studentischen Arbeitsgruppen. Entsprechende Lehrangebote sollten daher mehreren Studiengängen offenstehen, besonders attraktiv ist die Zusammenarbeit über Fachbereichs- und Fakultätsgrenzen hinweg. Idealerweise sollten die Kurse im Masterstudiengang oder zumindest gegen Ende des Bachelorstudiums angesiedelt sein, damit die Studierenden bereits Projekt- und Studienerfahrung mitbringen und sich auf die Besonderheit transformativ-transdisziplinärer Aufgabenstellungen konzentrieren können, statt z. B. mit dem Erwerb erster methodischer Kenntnisse beschäftigt zu sein.

Transformative und transdisziplinäre Lehre sind jedoch generell in vielen verschiedenen Settings, an verschiedenen Stellen eines Curriculums und an verschiedenen Hochschultypen (Universitäten, Hochschulen für Angewandte Wissenschaften, Duale Hochschulen) denkbar. Entsprechende Lehrprojekte oder Teile daraus lassen sich beispielsweise durchführen als
Interdisziplinäre

Zusammensetzung der Studierenden anstreben

Verschiedene Settings für transformative Lehre möglich 
Zweisemestriges Modul als Idealfall
- Ein- oder mehrsemestriges Wahlpflichtfach ${ }^{7}$

- Blockseminar ${ }^{8}$ mit wechselnden Präsenz- und Teamphasen

- Exkursion mit Basislager im Untersuchungsgebiet

- Projektseminar mit transdisziplinären Zusatzinhalten

- Online-Lehre mit Präsenzphase im Untersuchungsgebiet

- Online-Lehre mit hohem Interaktionsanteil zwischen Studierenden und Patinnen bzw. Paten

- Abschlussarbeit (Bachelor oder Master) mit zugrunde liegendem transformativem Projekt, das von einer Gruppe Studierender im Austausch mit Patinnen und Paten bearbeitet wird

Studierende besuchen in der Regel Lehrveranstaltungen nur einmal, selten über mehrere Semester hinweg. Das ist einerseits dem Interesse der Studierenden geschuldet, Einblicke in unterschiedliche Angebote zu erhalten, andererseits auch dem Curriculum, das den Besuch von Lehrangeboten nur bestimmten Semestern vorbehält. Ein Semester ist jedoch zu kurz, damit Studierende transformative Prozesse verstehen, das nötige Fach- und Nachhaltigkeitswissen erlangen, eine konkrete Problemstellung aus der Praxis im Co-Design mit Praxispartnern bearbeiten und diese in der Öffentlichkeit diskutieren können. In Kap. 5 stellen wir daher den idealtypischen Verlauf eines zweisemestrigen Moduls vor.

7 Wahlpflichtfach bedeutet, dass Studierende aus einem Lehrangebot ein Fach (oder mehrere Fächer) auswählen müssen.

8 Bei einer Blockveranstaltung wird der Lehrstoff nicht auf einzelne wöchentliche Termine im Semester verteilt, sondern in einem Block vermittelt, der z. B. aus zwei oder drei Wochen bestehen kann. 


\section{Konkrete Bausteine für die Lehre}

Das ist auch entscheidend, dass Lehre, die einen Praxisbezug aufweisen will, auch methodisch dahingehend arbeitet, dass da was Praxisnahes rauskommt.

Dr. Ralph Bürk, Digitalakademie Baden-Württemberg, WiNo-Projekt-Pate

In den vorangegangenen Kapiteln skizzierten wir die Grundlagen und Rahmenbedingungen der Zusammenarbeit mit Praxisakteuren im Kontext transformativer Lehre. Wie dieser Ansatz nun konkret umgesetzt werden kann, stellen wir im Folgenden anhand von 14 zentralen Bausteinen vor, die es Ihnen erleichtern sollen, Ihre eigenen Lehrabläufe zu planen. Wir zeigen dabei zum einen auf, wann und wie Praxisakteure in die Lehre integriert werden können, zum anderen, wie die Bearbeitung der studentischen Projekte begleitet bzw. angeleitet werden kann.

\subsection{Einordnung der Bausteine in die Phasen eines Projektes}

Unsere Bausteine für die Zusammenarbeit mit Praxisakteuren in der transformativen Lehre adressieren unterschiedliche Ziele und beinhalten verschiedene methodische Zugänge. In Anlehnung an die Phasen eines transdisziplinären Projektes ordnen wir sie den Phasen Co-Design, Co-Produktion, Co-Dissemination und Co-Evaluation $\mathrm{zu}^{9}$ (siehe Tab. 1). Hinzu kommt eine Rubrik, die

9 In der Literatur zu transdisziplinärer Forschung und Reallaboren allgemein üblich ist die Unterteilung in drei Phasen: 1) die Co-Design-Phase, die der Herausarbeitung des Forschungsprogramms im Austausch zwi-

Gestaltung transformativer Lehre mit Praxisanbindung 
phasenvorbereitende bzw. - unabhängige Ansätze enthält. Keine Erwähnung finden Aufgaben, die auch in anderen Lehrformen anstehen und zum allgemeinen Lehrmanagement gehören, wie z. B. die Einbindung der Lehrveranstaltungen in das Curriculum und die Bewerbung bei Studierenden oder die inhaltliche und didaktische Planung der Lehrveranstaltung.

schen Wissenschaft und Praxis dient, 2) die Co-Produktions-Phase, in der Problemlösungen und übertragbares Wissen von Wissenschaft und Praxis erarbeitet werden, 3) die Phase der Wissensintegration bzw. CoDissemination, in der die erarbeiteten Lösungen bzw. Wissensbestände in gesellschaftliche und wissenschaftliche Praktiken integriert werden (Bergmann \& Jahn 2005, Lang et al. 2012, Rhodius et al. 2016). In Rose et al. (2019) wird die dritte Phase als Co-Evaluation bezeichnet. Sie beinhaltet ebenso die Integration der Ergebnisse, stellt in ihrem Titel aber die gemeinsame Sichtung und Bewertung der Ergebnisse durch Wissenschaft und Praxis in den Vordergrund. Wir verwenden den Begriff der Co-Evaluation in diesem Leitfaden im Sinn des Evaluierens des Lehrmoduls bzw. Kurses sowie der transdisziplinären Zusammenarbeit. 
Tab. 1: Die Bausteine für die Zusammenarbeit mit Praxisakteuren in der transformativen Lehre

\begin{tabular}{|c|c|c|c|}
\hline \multirow{3}{*}{$\begin{array}{l}\text { Phase } \\
\text { Phasen- } \\
\text { vorberei- } \\
\text { tende bzw. } \\
\text {-unabhängige } \\
\text { Bausteine }\end{array}$} & \multicolumn{2}{|c|}{ Baustein } & \multirow{2}{*}{$\begin{array}{l}\text { Ziel } \\
\text { Praxisakteure für die Übernahme einer } \\
\text { Patenschaft gewinnen, Rollen und } \\
\text { Erwartungen klären }\end{array}$} \\
\hline & 1 & $\begin{array}{l}\text { Patinnen und Paten } \\
\text { gewinnen, Absprachen } \\
\text { treffen }\end{array}$ & \\
\hline & 2 & $\begin{array}{l}\text { Studierenden-Paten- } \\
\text { Austausch }\end{array}$ & $\begin{array}{l}\text { Die Kommunikation zwischen Patinnen } \\
\text { bzw. Paten und Studierenden organisieren, } \\
\text { Kommunikationskanäle und -zeitfenster } \\
\text { vereinbaren }\end{array}$ \\
\hline & 3 & $\begin{array}{l}\text { Team-Building- } \\
\text { Wochenende }\end{array}$ & $\begin{array}{l}\text { Den Teamgeist stärken, sich abseits vom } \\
\text { Alltag begegnen }\end{array}$ \\
\hline & 4 & $\begin{array}{l}\text { Kultur der } \\
\text { Zusammenarbeit fördern }\end{array}$ & $\begin{array}{l}\text { Studierende reflektieren ihre Rollen } \\
\text { in Gruppen und handeln Regeln der } \\
\text { Zusammenarbeit aus. }\end{array}$ \\
\hline & 5 & Peer-Feedback & $\begin{array}{l}\text { Studierende üben konstruktive Kritik an } \\
\text { den Projekten der anderen Teams und } \\
\text { entwickeln diese gemeinsam weiter. }\end{array}$ \\
\hline & 6 & $\begin{array}{l}\text { Projekt-Coaching durch } \\
\text { Lehrende }\end{array}$ & $\begin{array}{l}\text { Lernprozesse fördern, die Studierenden } \\
\text { durch Höhen und Tiefen der Projektarbeit } \\
\text { begleiten }\end{array}$ \\
\hline \multirow[t]{2}{*}{ Co-Design } & 7 & $\begin{array}{l}\text { In den Kontext der } \\
\text { Untersuchungsregion } \\
\text { bzw. Problemstellung } \\
\text { eintauchen }\end{array}$ & $\begin{array}{l}\text { Die Region bzw. Problemstellung } \\
\text { kennenlernen: Rahmenbedingungen und } \\
\text { aktuelle Herausforderungen }\end{array}$ \\
\hline & 8 & $\begin{array}{l}\text { Ein transformatives } \\
\text { Projekt planen }\end{array}$ & $\begin{array}{l}\text { Grundlagen transformativen und } \\
\text { transdisziplinären Arbeitens verstehen; } \\
\text { Methoden auswählen, Instrumente } \\
\text { entwickeln, Vorgehensplan entwickeln }\end{array}$ \\
\hline \multirow[t]{2}{*}{ Co-Produktion } & 9 & $\begin{array}{l}\text { Design-Thinking- } \\
\text { Workshop }\end{array}$ & $\begin{array}{l}\text { Prototypen zur Lösung von } \\
\text { Problemstellungen entwickeln }\end{array}$ \\
\hline & 10 & $\begin{array}{l}\text { Interventionen } \\
\text { durchführen und } \\
\text { bewerten }\end{array}$ & $\begin{array}{l}\text { Handlungsroutinen hinterfragen, } \\
\text { Wirkungen der Interventionen reflektieren }\end{array}$ \\
\hline \multirow[t]{2}{*}{$\begin{array}{l}\text { Co-Dissemi- } \\
\text { nation }\end{array}$} & 11 & Vor-Ort-Präsentation & $\begin{array}{l}\text { Lösungen präsentieren, mit Öffentlichkeit } \\
\text { teilen und diskutieren }\end{array}$ \\
\hline & 12 & $\begin{array}{l}\text { Ergebnisse im } \\
\text { Projektbericht festhalten }\end{array}$ & $\begin{array}{l}\text { Problemlösungen dokumentieren und die } \\
\text { Ergebnisse für andere nutzbar machen }\end{array}$ \\
\hline \multirow[t]{2}{*}{ Co-Evaluation } & 13 & $\begin{array}{l}\text { Befragung der } \\
\text { beteiligten Akteure }\end{array}$ & $\begin{array}{l}\text { Über Fragebogen, Interviews oder } \\
\text { Reflexion in der Gruppe das Feedback der } \\
\text { beteiligten Akteure einholen }\end{array}$ \\
\hline & 14 & S-P-L-Reflexion & $\begin{array}{l}\text { Die Ergebnisse der Befragung in einem } \\
\text { Workshop mit allen Beteiligten reflektieren }\end{array}$ \\
\hline
\end{tabular}


Tab. 2: Zuordnung der 14 Bausteine zu den Transformationskompetenzen nach Wiek et al. (2011)

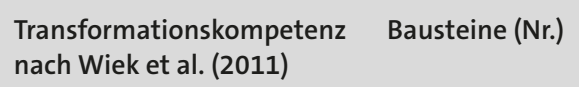

Systemisches Denken

Projekt-Coaching durch Lehrende (6) In den Kontext eintauchen (7) Ein transformatives Projekt planen (8) Interventionen durchführen und bewerten (10)

Vor-Ort-Diskussion der Ergebnisse (11)

Ergebnisse im Projektbericht festhalten (12)

Antizipative Kompetenz

Design-Thinking-Workshop (9) Interventionen durchführen und bewerten (10) Vor-Ort-Diskussion der Ergebnisse (11) Ergebnisse im Projektbericht festhalten (12)

Normative Kompetenz

Design-Thinking-Workshop (9)

Interventionen durchführen und bewerten (10)

Vor-Ort-Diskussion der Ergebnisse (11)

Ergebnisse im Projektbericht festhalten (12)

Strategische Kompetenz

Peer-Feedback (5)

Projekt-Coaching durch Lehrende (6)

Ein transformatives Projekt planen (8)

Interventionen durchführen und bewerten (10)

Vor-Ort-Diskussion der Ergebnisse (11)

Ergebnisse im Projektbericht festhalten (12)

Interpersonale Kompetenz

Studierenden-Paten-Austausch (2)

Teambuilding-Wochenende (3)

Kultur der Zusammenarbeit fördern (4)

Peer-Feedback (5)

Interventionen durchführen und bewerten (10)

Vor-Ort-Diskussion der Ergebnisse (11)

Ergebnisse im Projektbericht festhalten (12)

Befragung der beteiligten Akteure (13)

S-P-L-Reflexion (14) 


\subsection{Die Steckbriefe}

Die 14 Bausteine werden in einzelnen Steckbriefen beschrieben. Diese Steckbriefe enthalten die zum Verständnis der Bausteine nötigen Eckdaten, wie Ziele, Beteiligte, Ablauf, Zeitbedarf und Kosten. Sie geben zudem einen Einblick, welche Erfahrungen wir bei der Anwendung des Bausteins gemacht haben und welches Feedback Studierende oder Patinnen und Paten in der Evaluation (siehe Kap. 2.1) gaben. Einen beispielhaften Steckbrief finden Sie in Abb. 5.

\begin{tabular}{|c|c|}
\hline Steckbriefkategorie & $\begin{array}{l}\text { Welche Fragen werden in dieser Kategorie } \\
\text { beantwortet? }\end{array}$ \\
\hline Baustein mit Nr. & Wie lautet der Titel des Bausteins? \\
\hline Lehrkontext & $\begin{array}{l}\text { Wie ordnet sich der Baustein in den Lehrkontext } \\
\text { ein? }\end{array}$ \\
\hline Beteiligte & $\begin{array}{l}\text { Wer ist alles daran beteiligt? Wer hat die } \\
\text { Federführung? }\end{array}$ \\
\hline $\begin{array}{l}\text { Ziele/ } \\
\text { Kompetenzerwerb }\end{array}$ & $\begin{array}{l}\text { Welche Ziele werden mit dem Baustein verfolgt, } \\
\text { sowohl im Hinblick auf den Kompetenzerwerb } \\
\text { der Studierenden als auch in Bezug auf das } \\
\text { Erarbeiten praxisrelevanter Problemlösungen? }\end{array}$ \\
\hline $\begin{array}{l}\text { Beschreibung/ } \\
\text { Arbeitsschritte }\end{array}$ & $\begin{array}{l}\text { Wie verläuft so ein Baustein, ggf., welche } \\
\text { methodischen Elemente enthält er? }\end{array}$ \\
\hline Zeitbedarf & $\begin{array}{l}\text { Wie viel Zeit sollten Sie als Lehrende für die } \\
\text { Vorbereitung und Durchführung des Bausteins } \\
\text { einplanen? }\end{array}$ \\
\hline Kosten und Material & $\begin{array}{l}\text { Welches Material ist nötig, welche Kosten } \\
\text { können auf Sie zukommen? }\end{array}$ \\
\hline $\begin{array}{l}\text { Optionalität/ } \\
\text { Kombinierbarkeit }\end{array}$ & $\begin{array}{l}\text { Ist dieser Baustein zwingend erforderlich, } \\
\text { um transformative Lehre mit Praxisakteuren } \\
\text { durchführen zu können, oder ist er als } \\
\text { fakultativer Bestandteil zu sehen? Mit welchen } \\
\text { Bausteinen oder Methoden lässt er sich gut } \\
\text { kombinieren? }\end{array}$ \\
\hline Unsere Erfahrung & $\begin{array}{l}\text { Welche Erfahrungen haben wir mit diesem } \\
\text { Baustein gemacht, die wir an Sie weitergeben } \\
\text { möchten? }\end{array}$ \\
\hline $\begin{array}{l}\text { Erkenntnisse aus } \\
\text { der Evaluation }\end{array}$ & $\begin{array}{l}\text { Wie wurde der Baustein in der Evaluation durch } \\
\text { die Studierenden sowie die Patinnen und Paten } \\
\text { beurteilt? }\end{array}$ \\
\hline $\begin{array}{l}\text { Weiterführende } \\
\text { Literatur }\end{array}$ & $\begin{array}{l}\text { Gibt es empfehlenswerte weiterführende } \\
\text { Literatur? }\end{array}$ \\
\hline
\end{tabular}

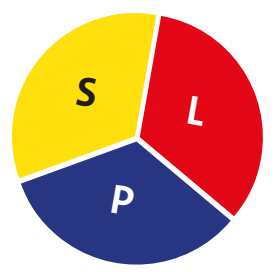

Die Kreisstücke zeigen an, wer an dem Baustein beteiligt ist. Wenn Akteure nur begleitend dabei sind, bleibt der jeweilige Kreissektor ohne Farbe.

$\mathrm{S}=$ Studierende

$\mathrm{P}=$ Patinnen und

Paten

$L=$ Lehrende

Abb. 4: Der Aufbau der Steckbriefe (eigene Darstellung) 
In Kap. 4.3 setzen wir aus den vorgestellten Bausteinen den aus unserer Sicht idealtypischen Verlauf eines zweisemestrigen Moduls transformativer Lehre zusammen. Da ein zeitlicher Rahmen von zwei Semestern jedoch eher selten zur Verfügung steht, möchten wir Sie zu einem flexiblen Umgang mit den Bausteinen ermutigen. Wählen Sie die Bausteine aus, die für Ihren Kontext passend sind. Zu Ihrer Orientierung haben wir in den Steckbriefen vermerkt, welche Bausteine wir für unverzichtbar für unseren Ansatz der Zusammenarbeit mit Praxisakteuren in der Lehre halten, und wie sich diese kombinieren lassen. 


\subsubsection{Phasenvorbereitende bzw. -unabhängige Bausteine}

\begin{tabular}{ll} 
Baustein 1 & $\begin{array}{l}\text { Praxispatinnen und -paten gewinnen, Absprachen } \\
\text { treffen }\end{array}$ \\
\hline Lehrkontext & • In Vorbereitung auf die Lehrveranstaltung \\
Beteiligte & - Lehrende und Praxisakteure \\
Ziele & - Akquise von Praxispatinnen und -paten und Projektthemen \\
& - Erstellen von Steckbriefen für die vorgesehenen Projekte \\
& - Klärung der Rollen und Erwartungen \\
& - Abstecken eines groben Zeit- und ggf. Budgetplans
\end{tabular}

Arbeitsschritte - Praxisakteure finden, die eigene Problemstellungen zur Bearbeitung durch die Studierenden einbringen oder bereit sind, die von den Lehrenden oder Studierenden eingebrachten Themen zu begleiten

- Informieren der Patinnen und Paten über die Ziele des Lehrformats, die Aufgaben der verschiedenen Akteure und die fachlichen Hintergründe der Studierenden, z. B. durch Steckbriefe zu den beteiligten Studiengängen

- Den zeitlichen Ablauf der wesentlichen Meilensteine des Moduls bzw. Kurses vor Start der Veranstaltung mit den Patinnen und Paten abstimmen

- Termine öffentlichkeitswirksamer Veranstaltungen außerhalb der Hochschule (z. B. Exkursion, Präsentation) frühzeitig in Absprache mit den Patinnen und Paten festlegen
Zeitbedarf $\quad$ Variabel, je nach eigener Vernetzung mit potenziellen Projektpatinnen und -paten, durchschnittlich 2 bis 3 Monate Vorlaufzeit
Kosten und Material
- Fahrtkosten für Studierende und/oder Patinnen und Paten, falls persönliche Besuche erforderlich sind
- Virtuelle Lehrplattform oder Team-Space für das Projekt hilfreich
Optionalität/ Kommbinier- barkeit
- Zwingende Voraussetzung für alle folgenden Bausteine und die Gewährleistung einer guten Zusammenarbeit
Unsere Erfahrungen
- Netzwerke, auf die Lehrende aus eigenen Projekten zurückgreifen können, erleichtern die Suche nach Praxisakteuren.
- Praxisakteure können keine sofort umsetzbaren "Auftragsarbeiten « von den Studierenden erwarten und investieren zudem einiges an Zeit. Sie sollten daher, neben dem Interesse an dem studentischen Projekt, Freude daran haben, junge Menschen als Mentorinnen bzw. Mentoren zu begleiten.
- Erfahrungsgemäß reicht die einmalige Absprache nicht aus; Erwartungen und Aufgaben müssen stattdessen durch wiederholte Kommunikation im Verlauf der Zusammenarbeit für alle präsent gehalten und ggf. angeglichen werden.

Erkenntnisse aus der - Der Vorschlag, den Patinnen und Paten Hintergrundinformationen über die Evaluation Studierenden und ihre bisherigen Kompetenzen zukommen zu lassen, geht auf Äußerungen der Studierenden in der Evaluation zurück. Diese hatten den Eindruck, dass die Patinnen und Paten höhere Erwartungen an sie hatten, als sie erfüllen konnten. 


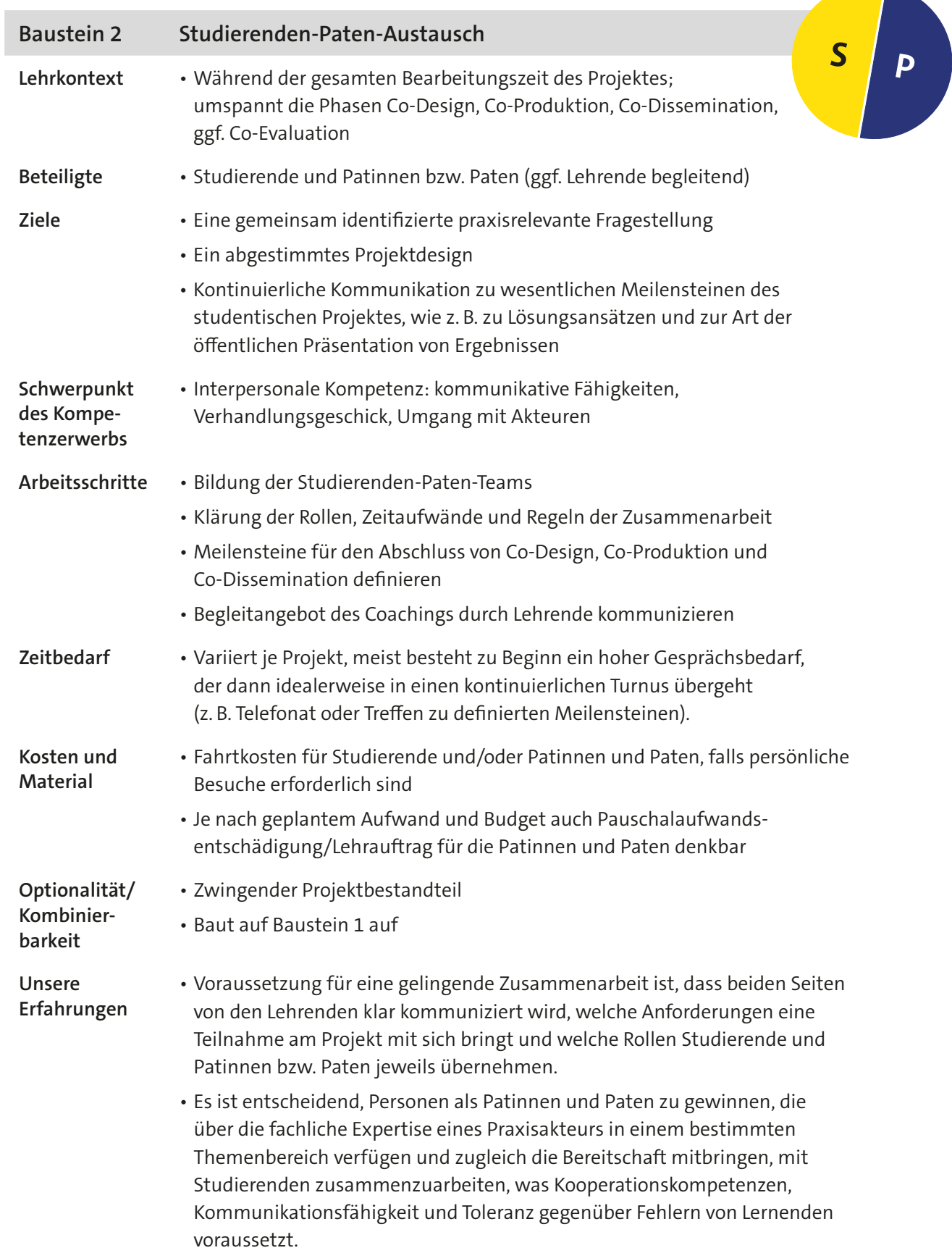


- Den Patinnen und Paten sollte frühzeitig kommuniziert werden, dass konfliktäre Situationen mit den Studierenden aufkommen könnten. Konflikte werden dabei als Teil des Lernprozesses für die Studierenden gesehen. Lehrende stehen, wenn notwendig, als vermittelnde Ansprechpersonen zur Verfügung.

- Die Organisation der Kommunikation zwischen Patinnen bzw. Paten und Studierenden ist ein Dreh- und Angelpunkt. Hier können die Lehrenden evtl. Vorgaben zur Häufigkeit der Kontaktaufnahme mit Patinnen und Paten durch die Studierenden machen. Auch kann innerhalb der Studierendengruppe eine Koordinatorin oder ein Koordinator benannt werden, die bzw. der für die Kommunikation mit der Patin oder dem Paten verantwortlich ist.

Erkenntnisse aus der Evaluation
Weiterführende Literatur
- Die Studierenden empfanden es als wertvolle Erfahrung, als gleichrangige Projektpartner wahrgenommen zu werden, wie folgender O-Ton aus einem Reflexions-Workshop an der Uni Freiburg belegt: »/ch dachte erst, die Praxispartner lächeln über uns Studis, aber die haben uns und unsere Ideen voll ernst genommen - fand ich gut."

- Die Patinnen und Paten leisteten wertvolle Beiträge zur Planung und Durchführung des Projektes. Die Studierenden lernten, auch mit kritischen Rückmeldungen der Patinnen und Paten umzugehen: "Das Interview mit dem Praxispartner hat zwar all unsere Pläne zerhauen, aber trotzdem war es gut. "Besonders hervorgehoben wurde der Umstand, dass das »Projekt nicht in der Schublade verstaubt", was oft bei anderen Projektarbeiten so empfunden wird. Durch die Einbindung ins Forschungsprojekt und den Austausch mit den Praxispatinnen und -paten war ein Rahmen gegeben, in dem die Arbeit der Studierenden genutzt und weiterverwendet werden konnte. Vereinzelt wurde allerdings auch die Kritik geäußert, dass Studierende nicht als "Dienstleister «ür die Zielerreichung von Unternehmen eingesetzt werden sollten.

- In einer quantitativen Evaluation der Lehre wurden ebenfalls Aspekte abgefragt, die die Zusammenarbeit zwischen Studierenden und Patinnen bzw. Paten betrafen. Die Aussage "ich habe ergebnisorientiert mit den Praxispartnern kommuniziert", bezeichneten $82 \%$ der Befragten $(n=66)$ als zutreffend. Rund $70 \%$ der Studierenden waren insgesamt mit ihrem Kompetenzzuwachs im Bereich Kommunikation eher zufrieden bis sehr zufrieden. Im Bereich der Kooperationskompetenz gaben 68,2\% der Befragten an, dass die "Kooperation mit den Praxispartnern für sie eine wertvolle Erfahrung war«. Zudem wurden die Studierenden ergänzend gefragt, ob es zutreffe, dass "der Austausch mit den Praxispartnerinnen und -partnern zu ihrem Lernprozess beigetragen hat". Hier stimmten 65,2 \% völlig oder eher zu.

- Hilger A, Keil A (2021) Education for sustainable development with transdisciplinary-oriented courses - experiences and recommendations for future collaborations in higher education teaching. Journal of Geography in Higher Education [DOI: 10.1080/03098265.2021.1946765] 


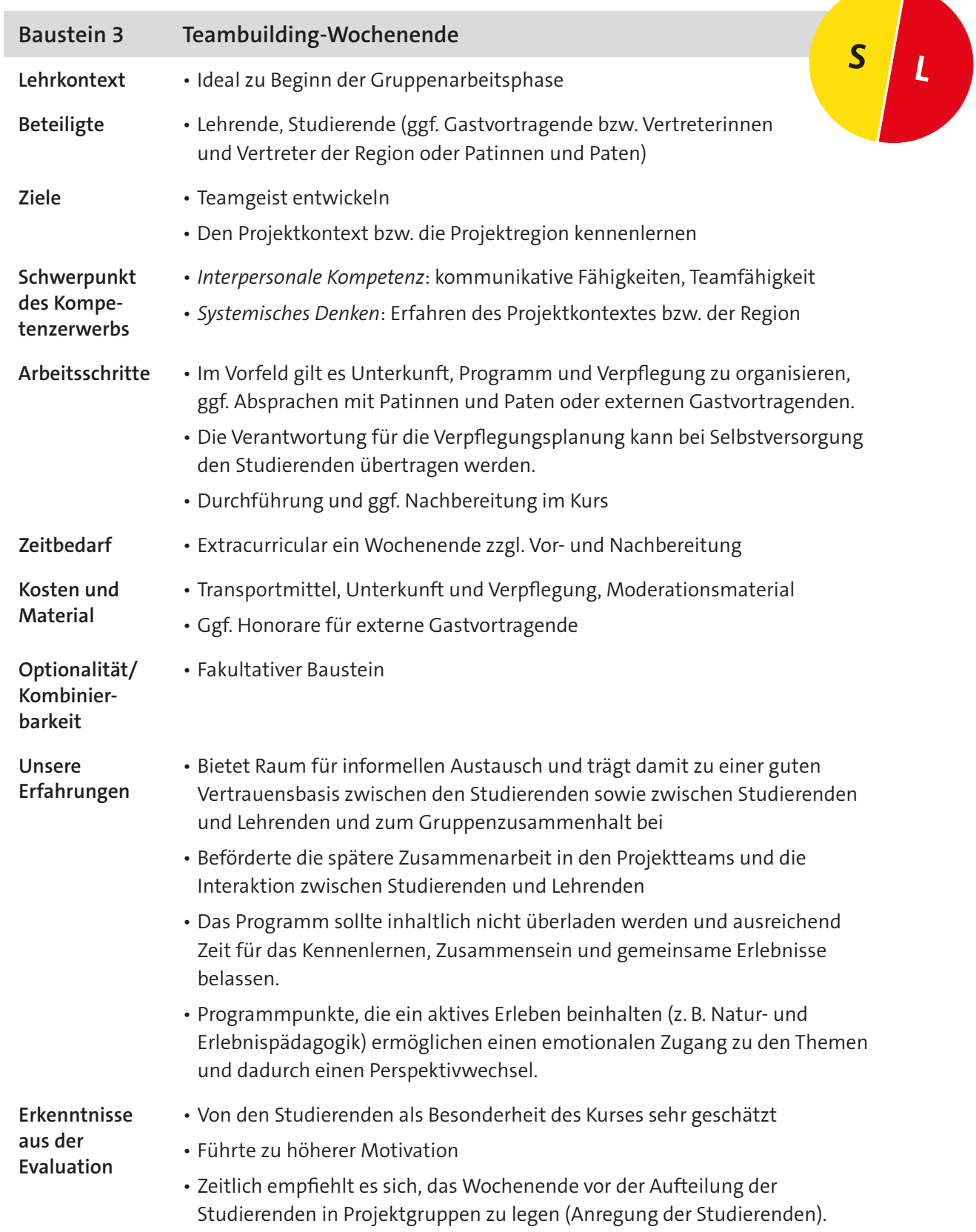




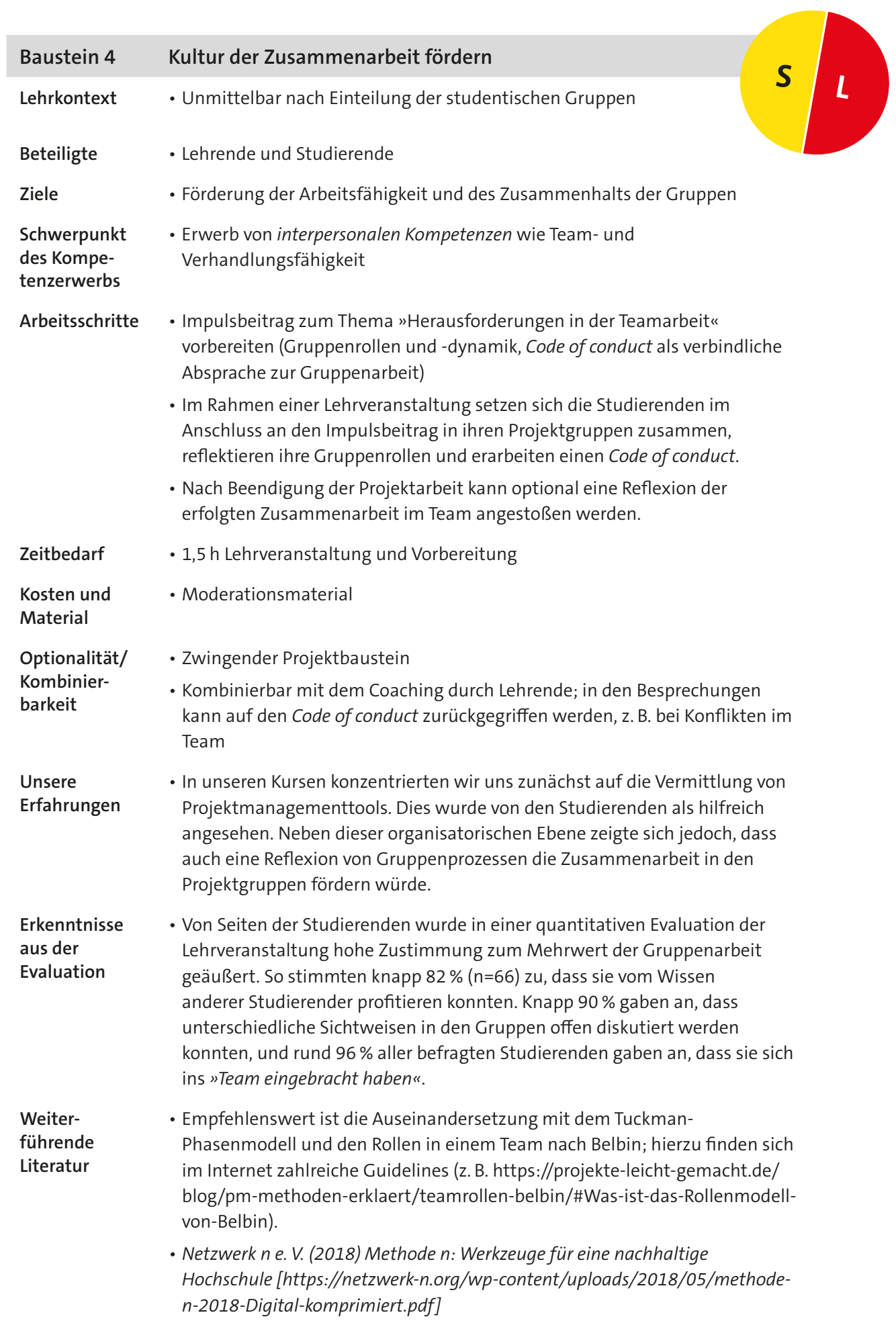




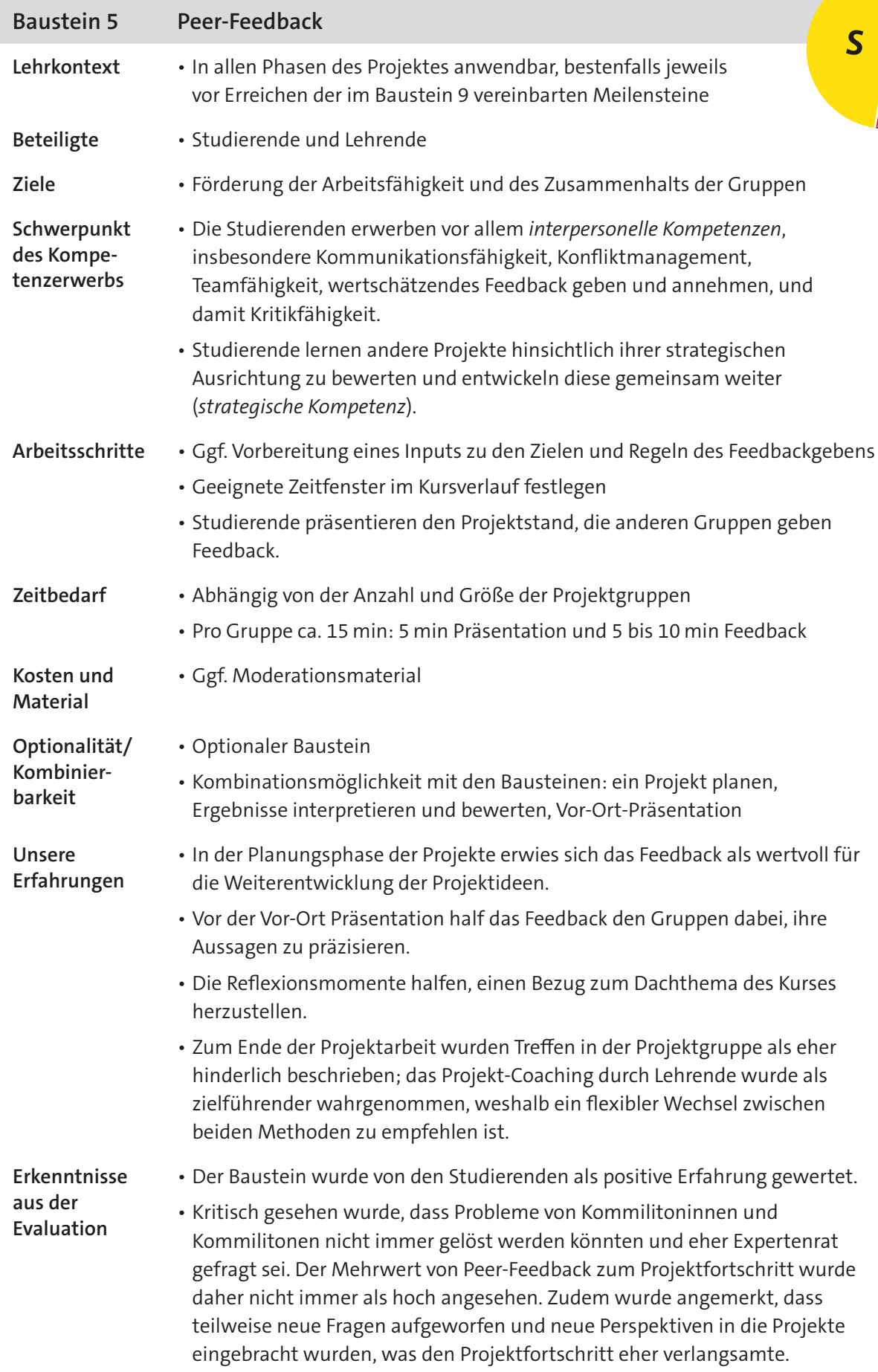

Arbeitsschritte - Ggf. Vorbereitung eines Inputs zu den Zielen und Regeln des Feedbackgebens

- Geeignete Zeitfenster im Kursverlauf festlegen

- Studierende präsentieren den Projektstand, die anderen Gruppen geben Feedback.

Zeitbedarf • Abhängig von der Anzahl und Größe der Projektgruppen

- Pro Gruppe ca. 15 min: 5 min Präsentation und 5 bis 10 min Feedback

Kosten und $\quad \cdot$ Ggf. Moderationsmaterial

Material

Optionalität/ • Optionaler Baustein

Kombinier-

barkeit

- Kombinationsmöglichkeit mit den Bausteinen: ein Projekt planen, Ergebnisse interpretieren und bewerten, Vor-Ort-Präsentation

Unsere

Erfahrungen

- In der Planungsphase der Projekte erwies sich das Feedback als wertvoll für die Weiterentwicklung der Projektideen.

- Vor der Vor-Ort Präsentation half das Feedback den Gruppen dabei, ihre Aussagen zu präzisieren.

- Die Reflexionsmomente halfen, einen Bezug zum Dachthema des Kurses herzustellen.

- Zum Ende der Projektarbeit wurden Treffen in der Projektgruppe als eher hinderlich beschrieben; das Projekt-Coaching durch Lehrende wurde als zielführender wahrgenommen, weshalb ein flexibler Wechsel zwischen beiden Methoden zu empfehlen ist.

Erkenntnisse aus der Evaluation

- Der Baustein wurde von den Studierenden als positive Erfahrung gewertet.

- Kritisch gesehen wurde, dass Probleme von Kommilitoninnen und Kommilitonen nicht immer gelöst werden könnten und eher Expertenrat gefragt sei. Der Mehrwert von Peer-Feedback zum Projektfortschritt wurde daher nicht immer als hoch angesehen. Zudem wurde angemerkt, dass teilweise neue Fragen aufgeworfen und neue Perspektiven in die Projekte eingebracht wurden, was den Projektfortschritt eher verlangsamte. 
Weiterführende Literatur
- Jürgens M, Golenia M (2020) E-Peer-Feedback zur Unterstützung Forschenden Lernens im Praxissemester - Konzept und Evaluationsergebnisse. In: Fischer B., Paul A. (Hg.) Lehren und Lernen mit und in digitalen Medien im Sport, CeBiS Schriftenreihe Vol. 18. Springer VS, Wiesbaden [http://doi-org-443.webvpn. jmu.edu.cn/10.1007/978-3-658-25524-4_9]

- Ditton H, Müller A (Hg.) (2014) Feedback und Rückmeldungen: Theoretische Grundlagen, empirische Befunde, praktische Anwendungsfelder. Waxmann, Münster [u.a.] 


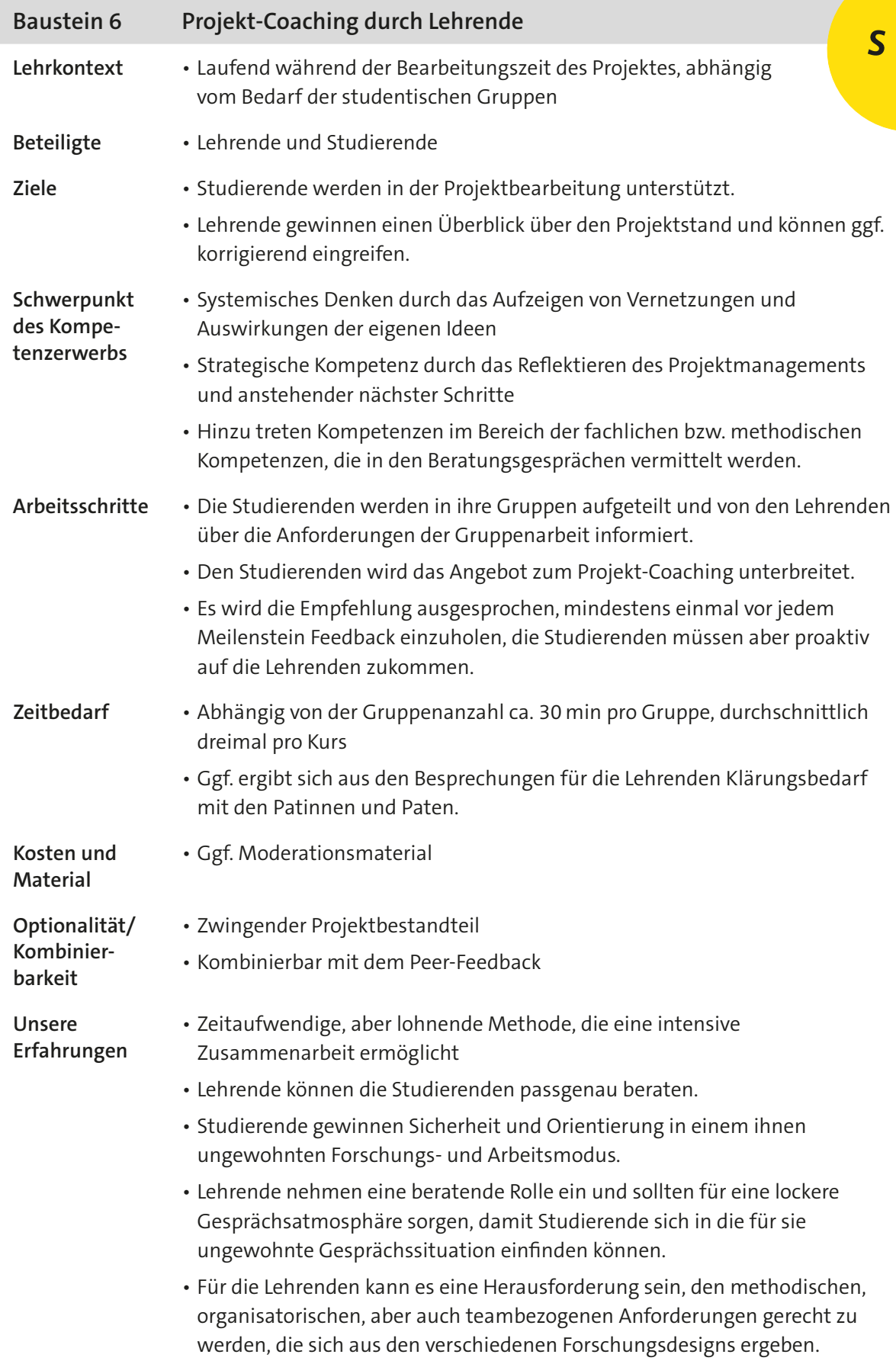

Arbeitsschritte - Die Studierenden werden in ihre Gruppen aufgeteilt und von den Lehrenden über die Anforderungen der Gruppenarbeit informiert.

- Den Studierenden wird das Angebot zum Projekt-Coaching unterbreitet.

- Es wird die Empfehlung ausgesprochen, mindestens einmal vor jedem Meilenstein Feedback einzuholen, die Studierenden müssen aber proaktiv auf die Lehrenden zukommen.

Zeitbedarf • Abhängig von der Gruppenanzahl ca. 30 min pro Gruppe, durchschnittlich dreimal pro Kurs

- Ggf. ergibt sich aus den Besprechungen für die Lehrenden Klärungsbedarf mit den Patinnen und Paten.

- Für die Lehrenden kann es eine Herausforderung sein, den methodischen, organisatorischen, aber auch teambezogenen Anforderungen gerecht zu werden, die sich aus den verschiedenen Forschungsdesigns ergeben. 
Erkenntnisse aus der Evaluation
- Das Coaching-Angebot wurde von den Studierenden stark genutzt und auch sehr positiv bewertet.

- Besonders wurde es geschätzt, wenn die Rückmeldungen seitens der Lehrenden sehr schnell erfolgten, da dadurch die nahtlose Weiterbearbeitung der Projekte möglich war.

- Durch die individuelle Betreuung konnten die Studierenden ihre methodischen Kompetenzen ausbauen. 


\subsubsection{Co-Design-Bausteine}

\begin{tabular}{|c|c|}
\hline Baustein 7 & $\begin{array}{l}\text { In den Kontext eintauchen: Auftaktveranstaltung, } \\
\text { Exkursion, Gastvortrag }\end{array}$ \\
\hline Lehrkontext & $\begin{array}{l}\text { - In der Co-Design-Phase, vor der Auswahl der Projektthemen durch } \\
\text { die Studierendengruppe }\end{array}$ \\
\hline Beteiligte & $\begin{array}{l}\text { - Lehrende, Studierende, Patinnen und Paten, Praxisakteure als } \\
\text { Gastvortragende }\end{array}$ \\
\hline Ziele & $\begin{array}{l}\text { - Studierende verstehen die Herausforderungen der Problemstellung aus der } \\
\text { Perspektive der Praxis. } \\
\text { - Der Besuch vor Ort verschafft einen emotionalen Zugang zum Thema. } \\
\text { - Studierende kommen in Kontakt mit ihren zukünftigen Patinnen und Paten. }\end{array}$ \\
\hline $\begin{array}{l}\text { Schwerpunkt } \\
\text { des } \\
\text { Kompetenz- } \\
\text { erwerbs }\end{array}$ & $\begin{array}{l}\text { - Die Studierenden erfahren die Komplexität der Fragestellungen und lernen, } \\
\text { vernetzt zu denken (systemisches Denken). }\end{array}$ \\
\hline \multirow[t]{5}{*}{ Beschreibung } & $\begin{array}{l}\text { - Der Baustein bringt die Studierenden in Kontakt mit der Region und mit } \\
\text { ihren Patinnen und Paten. Beides kann simultan erfolgen, beispielsweise im } \\
\text { Rahmen einer Exkursion mit Patinnen und Paten oder getrennt voneinander } \\
\text { wenn diese beispielsweise einen Gastvortrag halten und Studierende ohne } \\
\text { Patinnen und Paten die Untersuchungsregion erkunden. }\end{array}$ \\
\hline & $\begin{array}{l}\text { - Die drei Formate (Auftaktveranstaltung, Exkursion und Gastvorträge) } \\
\text { unterscheiden sich jedoch in ihrer Kontaktqualität: }\end{array}$ \\
\hline & $\begin{array}{l}\text { 1) Die geringste Kontaktqualität beinhaltet der Gastvortrag. Gastvorträge } \\
\text { können durch Patinnen und Paten oder andere Praxisakteure gegeben } \\
\text { werden. }\end{array}$ \\
\hline & $\begin{array}{l}\text { 2) Die Auftaktveranstaltung kann hingegen so durchgeführt werden, dass } \\
\text { sich Studierende und Patinnen bzw. Paten persönlich kennenlernen und } \\
\text { fachlich austauschen können (z. B. im Speed-Dating-Format). }\end{array}$ \\
\hline & $\begin{array}{l}\text { 3) Die Exkursion, wenn mit Patinnen und Paten durchgeführt, macht für die } \\
\text { Studierenden Praxisakteure in einem Praxisumfeld erlebbar. Sie ermöglicht } \\
\text { insofern eine hohe Kontaktqualität: } \\
\text { Durch direkte Kontakte mit der Untersuchungsregion, den dort relevanten } \\
\text { Fragestellungen, Stakeholdern und Rahmenbedingungen, vermittelt durch } \\
\text { die Patinnen und Paten, erhalten Studierende intensive Einblicke in die } \\
\text { Frage- und Problemstellungen in der Praxis. }\end{array}$ \\
\hline
\end{tabular}

\section{Arbeitsschritte Organisatorische Vorbereitung:}

- Auswahl der Untersuchungsregion und der Fragestellungen

- Festlegen der Formate (Auftaktveranstaltung und/oder Exkursion und/oder Gastvorträge)

- Abstimmung der Termine mit den Mitwirkenden

- Abstimmung der Örtlichkeiten und der Programminhalte der Veranstaltungen 


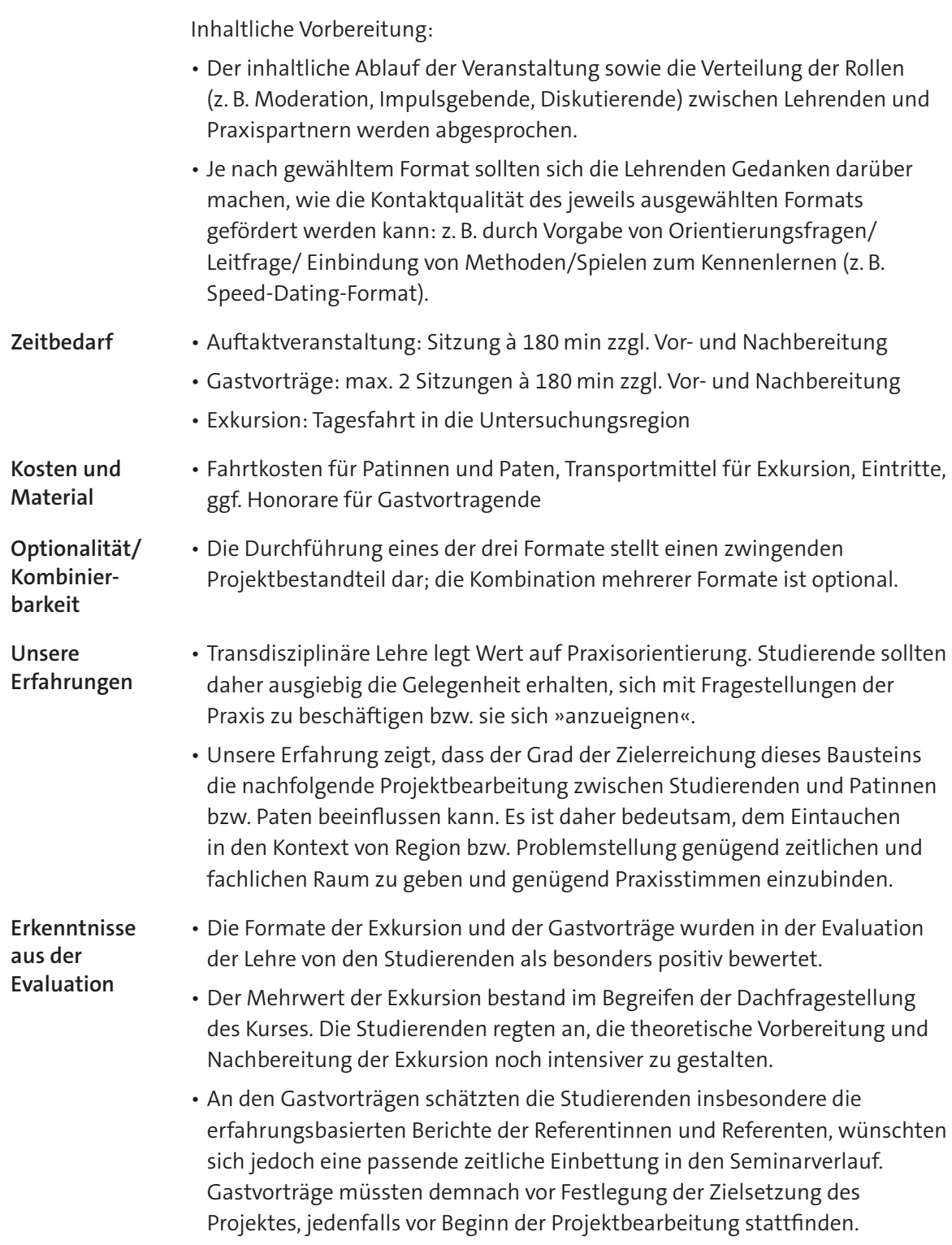




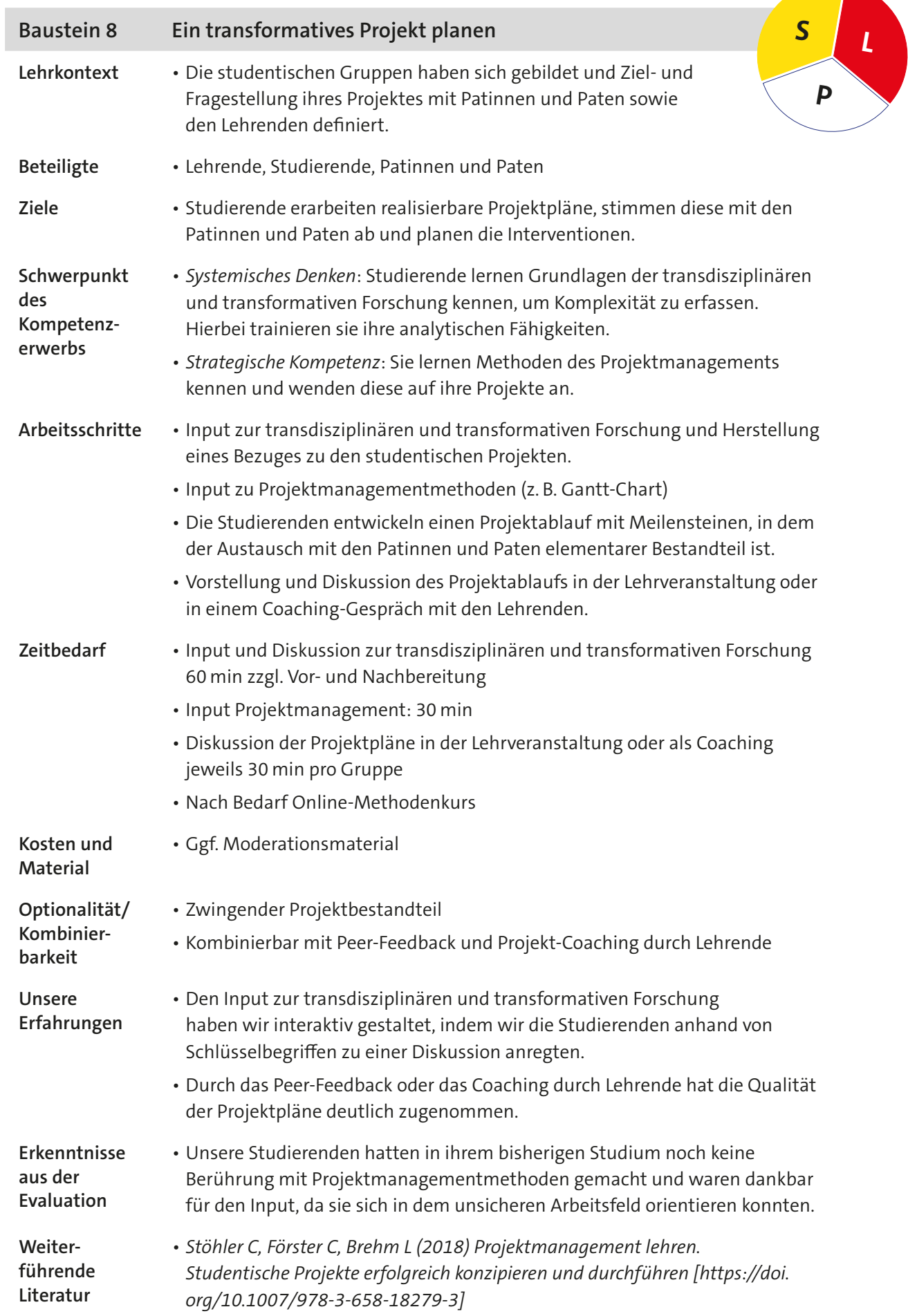




\subsubsection{Co-Produktions-Bausteine}

\begin{tabular}{|c|c|}
\hline Baustein 9 & Design-Thinking-Workshop \\
\hline Lehrkontext & $\begin{array}{l}\text { - Die studentischen Gruppen haben sich gebildet und Ziel- und } \\
\text { Fragestellung ihres Projektes mit Patinnen und Paten sowie } \\
\text { den Lehrenden definiert. }\end{array}$ \\
\hline Beteiligte & - Studierende, Lehrende, ggf. Patinnen und Paten \\
\hline Ziele & $\begin{array}{l}\text { - Entwicklung von Prototypen als kreativen Zugang zu Lösungen der } \\
\text { Problemstellung in einem gemeinsamen Workshop mit den Patinnen und } \\
\text { Paten }\end{array}$ \\
\hline \multirow{5}{*}{$\begin{array}{l}\text { Schwerpunkt } \\
\text { des } \\
\text { Kompetenz- } \\
\text { erwerbs }\end{array}$} & $\begin{array}{l}\text { - Systemisches Denken: Probleme ganzheitlich verstehen und aus } \\
\text { verschiedenen Perspektiven betrachten }\end{array}$ \\
\hline & - Antizipative Kompetenz: spielerische Entwicklung von Zukunftsvisionen \\
\hline & $\begin{array}{l}\text { - Normative Kompetenz: Auseinandersetzen mit den Werten und Normen der } \\
\text { Zielgruppe }\end{array}$ \\
\hline & $\begin{array}{l}\text { - Strategische Kompetenz: Erarbeitung von Strategien, um die Zielgruppe zu } \\
\text { adressieren }\end{array}$ \\
\hline & $\begin{array}{l}\text { - Interpersonale Kompetenz: Kommunikations- und Verhandlungsfähigkeit in } \\
\text { der (interdisziplinären) Gruppe und mit den Patinnen } \\
\text { und Paten }\end{array}$ \\
\hline \multirow[t]{4}{*}{ Arbeitsschritte } & $\begin{array}{l}\text { - Wenn Patinnen und Paten teilnehmen sollten, ist eine frühzeitige } \\
\text { Terminvereinbarung mit diesen nötig. }\end{array}$ \\
\hline & $\begin{array}{l}\text { - Programm und Zielsetzung des Workshops gegenüber den Studierenden } \\
\text { und den Patinnen und Paten kommunizieren }\end{array}$ \\
\hline & $\begin{array}{l}\text { - Raum und Materialien organisieren (besondere Anforderungen an } \\
\text { Raumgestaltung und Arbeitsmaterialien, siehe Literatur unten) }\end{array}$ \\
\hline & - Workshopergebnisse dokumentieren \\
\hline Zeitbedarf & - Halber oder ganzer Tag zzgl. Vor- und Nachbereitung \\
\hline \multirow[t]{4}{*}{$\begin{array}{l}\text { Kosten und } \\
\text { Material }\end{array}$} & $\begin{array}{l}\text { - Sachmittel (z. B. Moderationsmaterial, großformatige Drucke, Materialien für } \\
\text { Prototypenbau) }\end{array}$ \\
\hline & - Ggf. Raummiete und Bewirtung \\
\hline & $\begin{array}{l}\text { - Ggf. Anfahrtskosten für Patinnen und Paten, eventuell auch für Studierende, } \\
\text { wenn hochschulexterner Ort }\end{array}$ \\
\hline & - Ggf. Honorar für externe Moderation \\
\hline $\begin{array}{l}\text { Optionalität/ } \\
\text { Kombinier- } \\
\text { barkeit }\end{array}$ & $\begin{array}{l}\text { - Optionaler Baustein, kombinierbar mit Baustein »Interventionen } \\
\text { durchführen und bewerten« }\end{array}$ \\
\hline
\end{tabular}


Unsere - Das Workshopformat wurde zunächst im Rahmen eines Expertenworkshops

Erfahrungen zur Generierung von Projektideen angewendet; die Ideen wurden als Steckbriefe festgehalten und dienten dann als Aufgabenstellung für die Studierendengruppen.

- Innerhalb des weiteren Projektverlaufs wurden Workshops ausschließlich mit den Studierenden durchgeführt. Das Problem bestand hierbei darin, im Nachgang passende Patinnen bzw. Paten für die entworfenen Projektideen zu finden; die Durchführung von Design Thinking in gemischten Studierenden-Paten-Gruppen erscheint daher als die bessere Lösung.

Erkenntnisse aus der Evaluation

Weiterführende Literatur
- Design Thinking wurde von Studierenden als geeignetes Instrument zum Erarbeiten von Lösungsansätzen empfunden. Zur Wahl des Zeitpunktes: Er sollte nicht zu früh liegen, damit die Studierenden Zeit haben, sich mit der Region und deren Fragestellungen auseinanderzusetzen und sich als Team zu finden; andererseits darf er nicht zu spät liegen, da die Projektideen dann bereits zu konkret sind und die notwendige Offenheit für kreatives Arbeiten dann nicht mehr gegeben ist.

- Als Kritik wurde geäußert, dass in den Design-Prozessen Projekte definiert wurden, die sehr unterschiedlich in ihrer Größe und Komplexität waren; damit verbundene Arbeitsaufwände waren nicht vergleichbar. Daher sind die Themen, die die Studierenden bearbeiten, vergleichbar und realistisch zu fassen, um für alle zufriedenstellende Ergebnisse zu erzielen.

- Uebernickel F, Brenner W, Naef T, Pukall B, Schindlholzer B (2015) Design Thinking: Das Handbuch. Frankfurter Allgemeine Buch, Frankfurt 


$\begin{array}{ll}\text { Baustein } 10 & \text { Interventionen durchführen und bewerten } \\ \text { Lehrkontext } & \text { • Im Anschluss an die Projektplanung } \\ \text { Beteiligte } & \text { • Studierende, begleitend Lehrende und Patinnen bzw. Paten } \\ \text { Ziele } & \text { • Impulse setzen für nachhaltiges Denken und Handeln } \\ & \text { - Studierende erfahren, dass Interventionen einen Veränderungs- und } \\ & \text { Diskussionsraum schaffen können, sowie die Grenzen von Inventionen. } \\ \text { Schwerpunkt } & \text { - Systemisches Denken: Die Studierenden ordnen ihre Intervention in größere } \\ \text { des } & \text { Zusammenhänge ein. } \\ \text { Kompetenz- } & \text { - Antizipative Kompetenz: Die Interventionen erfolgen im Hinblick auf eine } \\ \text { erwerbs } & \text { angestrebte Zukunft. } \\ & \text { - Normative Kompetenz: Durch Interventionen werden gültige Normen und } \\ & \text { Werte gezielt hinterfragt. } \\ & \text { Strategische Kompetenz: Die Durchführung der Interventionen erfordert } \\ & \text { strategische Planung und Vorbereitung. } \\ & \text { Interpersonale Kompetenz: Studierende verlassen ihre "Komfortzone« und } \\ \text { den gewohnten universitären Kontext. Sie konfrontieren sich dabei mit } \\ \text { anderen Wirklichkeiten und reflektieren ihr eigenes Handeln. }\end{array}$

Arbeitsschritte Da sich die Interventionen in ihrer Art stark voneinander unterscheiden (siehe Kap. 1), kann hier nur eine grobe Darstellung der Schritte erfolgen:

- Vorbereitung der Intervention (ggf. Materialbesorgung); hier erfolgt auch bereits die Planung der Auswertung (z. B. über Methoden der empirischen Sozialforschung)

- Durchführung der Interventionen

- Auswertung der Interventionen

Zeitbedarf • Je nach Intervention kann deren Durchführung punktuell sein oder sich über einen Monat erstrecken.

Kosten und - Material nach Bedarf, Fahrtkosten für die Studierenden, ggf. auch für die Material Patinnen und Paten

Optionalität/ • Zwingender Projektbestandteil

Kombinier-

barkeit

Unsere

Erfahrungen

- Die Wahrscheinlichkeit, dass die Projektideen von Patinnen und Paten weitergeführt werden, steigt, wenn sie vorher erprobt wurden.

- Die Arbeit mit den Studierenden ermöglicht den Patinnen und Paten, experimentell Themen zu bearbeiten und Lösungsansätze zu testen, die in den engen Grenzen ihrer Institutionen wenig Raum haben.

- Die Lehrenden sollten jedoch darauf achten, dass Studierende nicht für Interessen der Patinnen und Paten instrumentalisiert oder durch die Bearbeitung konfliktbehafteter Themen überfordert werden. 
Erkenntnisse aus der

Evaluation

Weiter-

führende Literatur
- Die Patinnen und Paten bezeichneten die Zusammenarbeit mit den Studierenden als inspirierend und erfrischend.

- Vereinzelt gab es kritische Stimmen bezüglich der Realisierbarkeit der Ideen.

- Die Studierenden hatten das Gefühl, Impulse setzen zu können, die tatsächlich Veränderungen bewirken können.

- West C (2018) "Wissen to Go" - Transdisziplinär-transformative Lehre als "Reallabor im Kleinen". In: Di Giulio A, Defila R (Hg.) Transdisziplinär und transformativ forschen. Eine Methodensammlung. Springer VS, Wiesbaden, S. 329-373

- Quartier Zukunft (Hg.) (2020): Dein Quartier und Du Nachhaltigkeitsexperimente im Reallabor zu Nachbarschaften, Bienen, Naschbeeten, Kreativität und Konsum [DOI: 10.5445/KSP/1000076132]

- Reallabor Nachhaltige Mobilität (Hg.) (2018): Die Kultur des Experimentierens. In Reallaboren Nachhaltigkeit gemeinsam schaffen [http://www.r-n-m.net/ wp-content/uploads/2018/03/Forschen-mit-Realexperimenten.pdf] 


\subsubsection{Co-Disseminations-Bausteine}

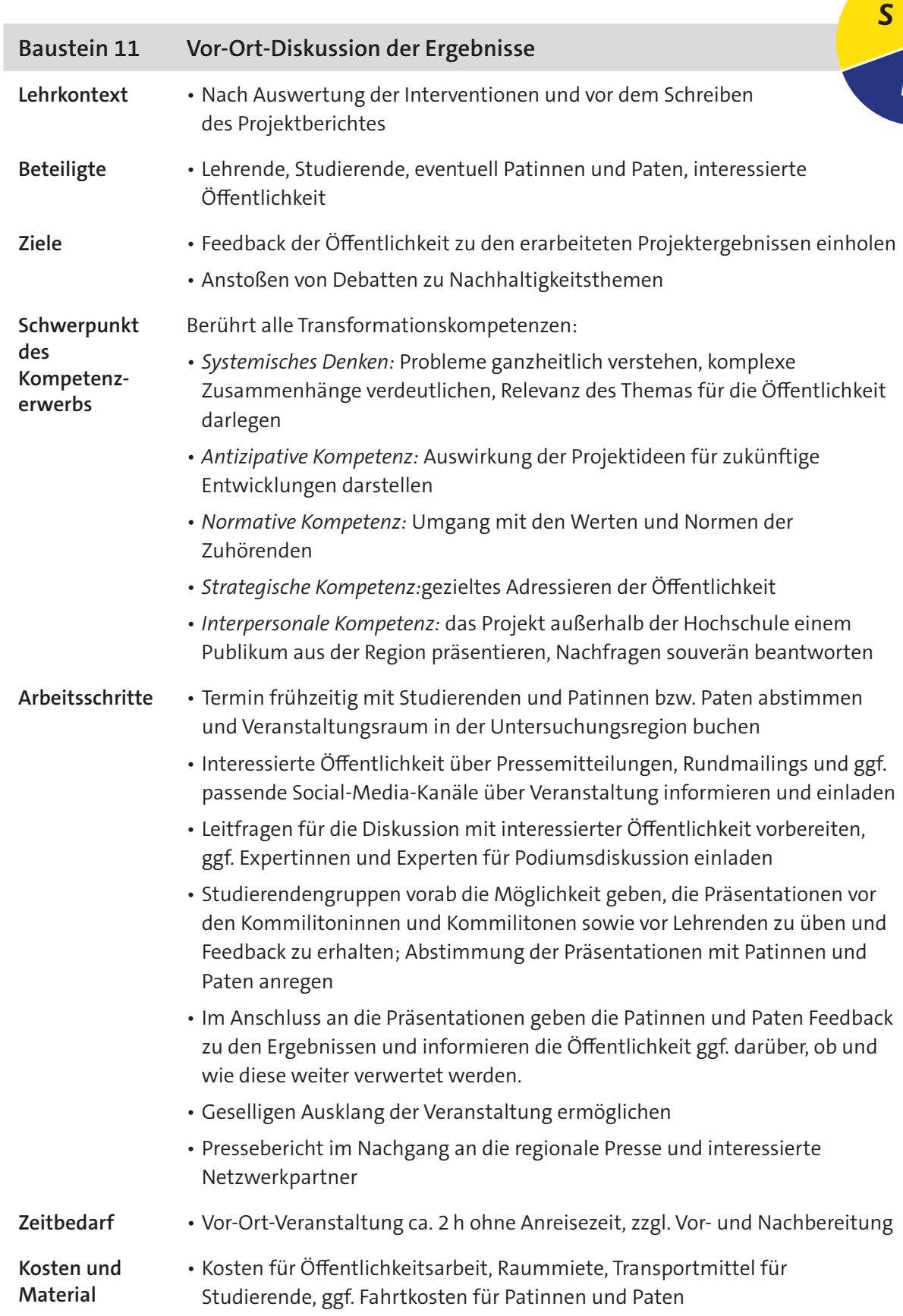


Optionalität/ • Zwingender Projektbaustein, kombinierbar mit Peer-Feedback und KombinierProjektbericht

barkeit

Unsere

Erfahrungen

- Die Vor-Ort-Präsentationen erwiesen sich als ein Herzstück der Lehre: Für die Studierenden war es eine wertvolle Erfahrung, vor einem öffentlichen Publikum zu präsentieren und ganz unmittelbare Rückmeldungen zu ihren Ideen zu erhalten. Immer wieder erstaunlich war, mit welcher Offenheit die Zuhörerschaft auf die Ideen der Studierenden einging; das Format ist daher sehr geeignet, um die öffentliche Debatte auch zu kontroversen Themen anzustoßen.

- Zu beachten ist, dass die Vor-Ort-Veranstaltung etwas Vorlauf vor Semesterende haben sollte - zum einen, damit die Anregungen aus dem Publikum noch in die studentischen Projektberichte einfließen können, zum anderen, um eine zu hohe Belastung für die Studierenden zu vermeiden.

- Ein geselliger Ausklang mit Snacks und Getränken ist zu empfehlen, um Netzwerken und Austausch zu ermöglichen; ein solcher Ausklang wird von den Studierenden und den Patinnen und Paten als sehr wertschätzend erlebt.

Erkenntnisse

- Als wesentlicher Mehrwert wurde von den Studierenden der Erwerb von

aus der

Evaluation sozialen Kompetenzen, von Sicherheit im Umgang mit Öffentlichkeit und im Vortrag dargestellt: "Präsentieren außerhalb der Uni ist gut, auch, dass Praxispartner dabei sind - da gibt man sich viel mehr Mühe."

- In der Evaluation konnten die Teilnehmenden angeben, ob sie ihre Ergebnisse praxisrelevant dargestellt haben; $94 \%(n=66)$ stimmten dieser Aussage zu. 


\begin{tabular}{|c|c|}
\hline Baustein 12 & Ergebnisse im Projektbericht festhalten \\
\hline Lehrkontext & - Im Anschluss an die Vor-Ort-Präsentation \\
\hline Beteiligte & $\begin{array}{l}\text { - Studierende, ggf. in Rücksprache mit Lehrenden und Patinnen } \\
\text { bzw. Paten }\end{array}$ \\
\hline \multirow[t]{2}{*}{ Ziele } & $\begin{array}{l}\text { - Studierende dokumentieren ihre Zwischenergebnisse so, dass die Patinnen } \\
\text { und Paten bzw. weitere Praxisakteure gut daran anschließen können. }\end{array}$ \\
\hline & $\begin{array}{l}\text { - Mit dem Projektbericht liegt zugleich ein Ergebnis vor, das Projektverlauf } \\
\text { und Kompetenzzuwachs der Studierenden dokumentiert. }\end{array}$ \\
\hline
\end{tabular}
Schwerpunkt Berührt alle Transformationskompetenzen:
des
Kompetenz- erwerbs
- Systemisches Denken: Probleme ganzheitlich verstehen, komplexe Zusammenhänge verdeutlichen, Relevanz des Themas für die Gesellschaft darlegen
- Antizipative Kompetenz: Auswirkung der Projektideen für zukünftige Entwicklungen darstellen
- Normative Kompetenz: Umgang mit widerstreitenden Werten und Normen verdeutlichen
- Strategische Kompetenz: gezieltes Adressieren der Leserschaft
- Interpersonale Kompetenz: Projektergebnisse im Team interpretieren

Arbeitsschritte • Die Anforderungen an den Projektbericht (Inhalte, Umfang) frühzeitig an die Studierenden kommunizieren und ggf. im Coaching-Termin offene Fragen besprechen

- Mit den Patinnen und Paten vorab abstimmen, welche Elemente der Projektbericht enthalten sollte, um für sie umsetzungsrelevant zu sein

- Das auf der Vor-Ort-Präsentation von den Zuhörenden erhaltene Feedback sollte von den Studierenden reflektiert werden.

Zeitbedarf - Abstimmungsprozesse mit Patinnen bzw. Paten und Studierenden sowie Betreuungsaufwand im Rahmen der Coaching-Gespräche

- Bewertung bei ausführlichem Feedback an die Studierenden dauert ca. $2 \mathrm{~h}$ pro Projektskizze

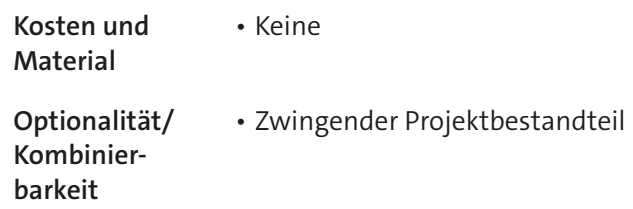


Unsere

Erfahrungen

Erkenntnisse

aus der

Evaluation
- Die Projektskizze wurde von den Studierenden oft mit großem Engagement verfasst. In den ersten Kursen erfolgte noch keine Abstimmung der Inhalte mit den Patinnen und Paten. Dies führte zu Informationsverlusten und erschwerte die Übernahme der Projektideen durch die Patinnen und Paten. Daher gingen wir dazu über, die nötigen Angaben des Berichts vorab mit den Patinnen und Paten abzustimmen.

- Der Umfang des Projektberichts sollte überschaubar gehalten werden (z. B. max. 10 Seiten), da die Studierenden im Kurs bereits ein hohes Arbeitspensum bewältigen.

- Die Studierenden empfanden es als sehr motivierend, dass ihre Projektskizzen als Grundlage für die weitere Bearbeitung und Umsetzung ihrer Ideen dienten. 


\subsubsection{Co-Evaluations-Bausteine}

\begin{tabular}{|c|c|}
\hline Baustein 13 & Befragung der beteiligten Akteure \\
\hline Lehrkontext & - Am Ende des Kurses bzw. Moduls \\
\hline Beteiligte & - Lehrende, Studierende, Patinnen und Paten \\
\hline \multirow[t]{3}{*}{ Ziele } & $\begin{array}{l}\text { - Prüfen, inwieweit die Studierenden die angestrebten Kompetenzziele } \\
\text { tatsächlich erreichen konnten }\end{array}$ \\
\hline & $\begin{array}{l}\text { - Zufriedenheit der Patinnen und Paten mit der Zusammenarbeit mit } \\
\text { Studierenden/Lehrenden sowie mit den Ergebnissen erfahren }\end{array}$ \\
\hline & • Hinweise für die Gestaltung zukünftiger Kurse/Module erhalten \\
\hline $\begin{array}{l}\text { Schwerpunkt } \\
\text { des } \\
\text { Kompetenz- } \\
\text { erwerbs }\end{array}$ & $\begin{array}{l}\text { - Interpersonale Kompetenz durch das Reflektieren des eigenen } \\
\text { Kompetenzzuwachses }\end{array}$ \\
\hline \multirow[t]{5}{*}{ Arbeitsschritte } & $\begin{array}{l}\text { - Vorbereitung eines auf dem angestrebten Kompetenzerwerb aufbauenden } \\
\text { Fragebogens für die Studierenden }\end{array}$ \\
\hline & $\begin{array}{l}\text { - Offenes Reflexionsgespräch mit den Studierenden am Ende des Kurses und } \\
\text { Verteilung des Evaluierungsbogens }\end{array}$ \\
\hline & - Auswertung des Evaluierungsbogens \\
\hline & $\begin{array}{l}\text { - Vorbereitung, Durchführung und Auswertung von Reflexionsgesprächen mit } \\
\text { den Patinnen und Paten }\end{array}$ \\
\hline & - Selbstreflexion der Lehrenden, inwieweit die Lehrziele erreicht wurden \\
\hline Zeitbedarf & $\begin{array}{l}\text { •Insgesamt sehr hoch; wenn möglich, auf personelle Unterstützung } \\
\text { durch Fachleute aus der Hochschuldidaktik (Hilfe bei der Erstellung des } \\
\text { Fragebogens) oder studentische Hilfskräfte (Auswertung des Fragebogens) } \\
\text { zurückgreifen }\end{array}$ \\
\hline $\begin{array}{l}\text { Kosten und } \\
\text { Material }\end{array}$ & - Materialkosten, Kosten für studentische Hilfskraft \\
\hline $\begin{array}{l}\text { Optionalität/ } \\
\text { Kombinier- } \\
\text { barkeit }\end{array}$ & $\begin{array}{l}\text { - Optionaler Baustein; wenn dieser nicht stattfindet, sollte der Baustein } \\
\text { Reflexionsworkshop der beteiligten Akteure stattfinden }\end{array}$ \\
\hline $\begin{array}{l}\text { Unsere } \\
\text { Erfahrungen }\end{array}$ & $\begin{array}{l}\text { - Die Durchführung der Befragungen war nur infolge der Einbindung in ein } \\
\text { gefördertes Projekt möglich. }\end{array}$ \\
\hline & $\begin{array}{l}\text { - Da die Erstellung eines Lehrkonzeptes zu unseren Aufgaben gehörte, hatten } \\
\text { wir Raum für die ausführliche Auswertung und Selbstreflexion. }\end{array}$ \\
\hline $\begin{array}{l}\text { Erkenntnisse } \\
\text { aus der } \\
\text { Evaluation }\end{array}$ & $\begin{array}{l}\text { - Für Patinnen und Paten ist das Format eines Reflexionsgespräches gut } \\
\text { geeignet und wird als echtes Interesse an der Weiterentwicklung des } \\
\text { Lehrformats empfunden. }\end{array}$ \\
\hline & $\begin{array}{l}\text { - Die Kombination aus Fragebogen und Reflexionsgespräch zur Erfassung des } \\
\text { Studierendenfeedbacks ermöglicht sowohl Vertraulichkeit als auch direkten } \\
\text { Austausch. }\end{array}$ \\
\hline
\end{tabular}




\section{Konkrete Bausteine für die Lehre}

Weiter- - Zentrum für Hochschul- und Qualitätsentwicklung an der Universität führende Literatur Duisburg-Essen (2014) Diversität konkret - Handreichung für das Lehren und Lernen an Hochschulen, Feedback-Methodenbar. [https://duepublico2. uni-due.de/servlets/MCRFileNodeServlet/duepublico_derivate_00036794/07_ Feedback_Methodenbar.pdf] 


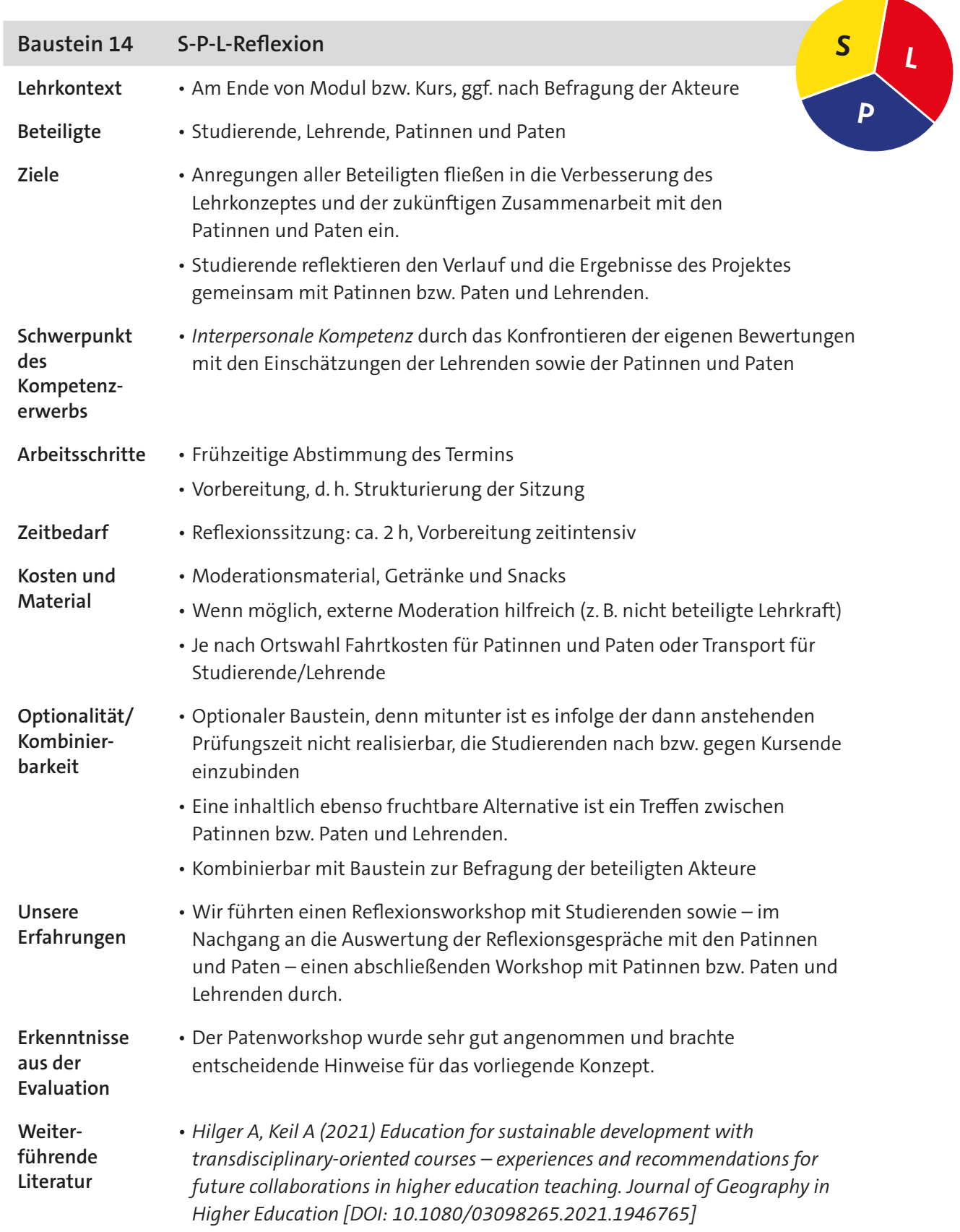




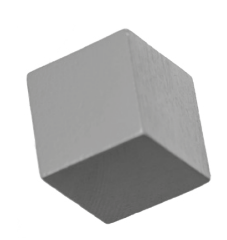

\title{
5. Und so könnte ein gesamtes Modul aussehen
}

\author{
Ich dachte erst, die Praxispartner lächeln über uns Studis, aber die haben \\ uns und unsere Ideen voll ernst genommen - fand ich gut. \\ Anonymes Feedback einer/eines \\ Studierenden in der Evaluierung
}

\begin{abstract}
Für ein weiterführendes Lehrkonzept muss sich die Hochschule im Klaren darüber sein, was sie von den Studierenden fordert, und sich fragen, ob sie genug Zeit einräumt, damit die Studierenden das dann auch wirklich gut umsetzen können.

Olfert Dorka, Garten- und Landschaftsarchitekt \& Landschaftstherapeut, WiNo-Projekt-Pate
\end{abstract}

Nachdem wir Ihnen im vorangegangenen Kapitel verschiedene Bausteine der Zusammenarbeit mit Praxisakteuren in der transformativen Lehre vorgestellt haben, möchten wir diese nun zu einem idealtypischen Modul zusammenfügen. Ein solches Modul beinhaltet zwei Semester. Es besteht aus 240 Unterrichtseinheiten (120 UE Präsenz, 120 UE Eigenleistung) und wird mit 6 ECTS bewertet. Je nach den vorliegenden Rahmenbedingungen kann dieser Arbeitsaufwand innerhalb von zwei folgenden Semestern erbracht werden oder in zwei zeitlich getrennten Semestern (z. B. jeweils Sommersemester). Abbildung 5 zeigt auf, wie sich die einzelnen Bausteine und Phasen auf diese zwei Semester verteilen. Die phasenvorbereitenden bzw. phasenunabhängigen Bausteine sind gesondert dargestellt und entweder im Vorfeld des Semesterbeginns einzuplanen oder je nach Bedarf einzelnen Phasen und Bausteinen zuzuordnen. Hinweise auf den geeigneten Zeitpunkt finden sich in den jeweiligen Steckbriefen. 


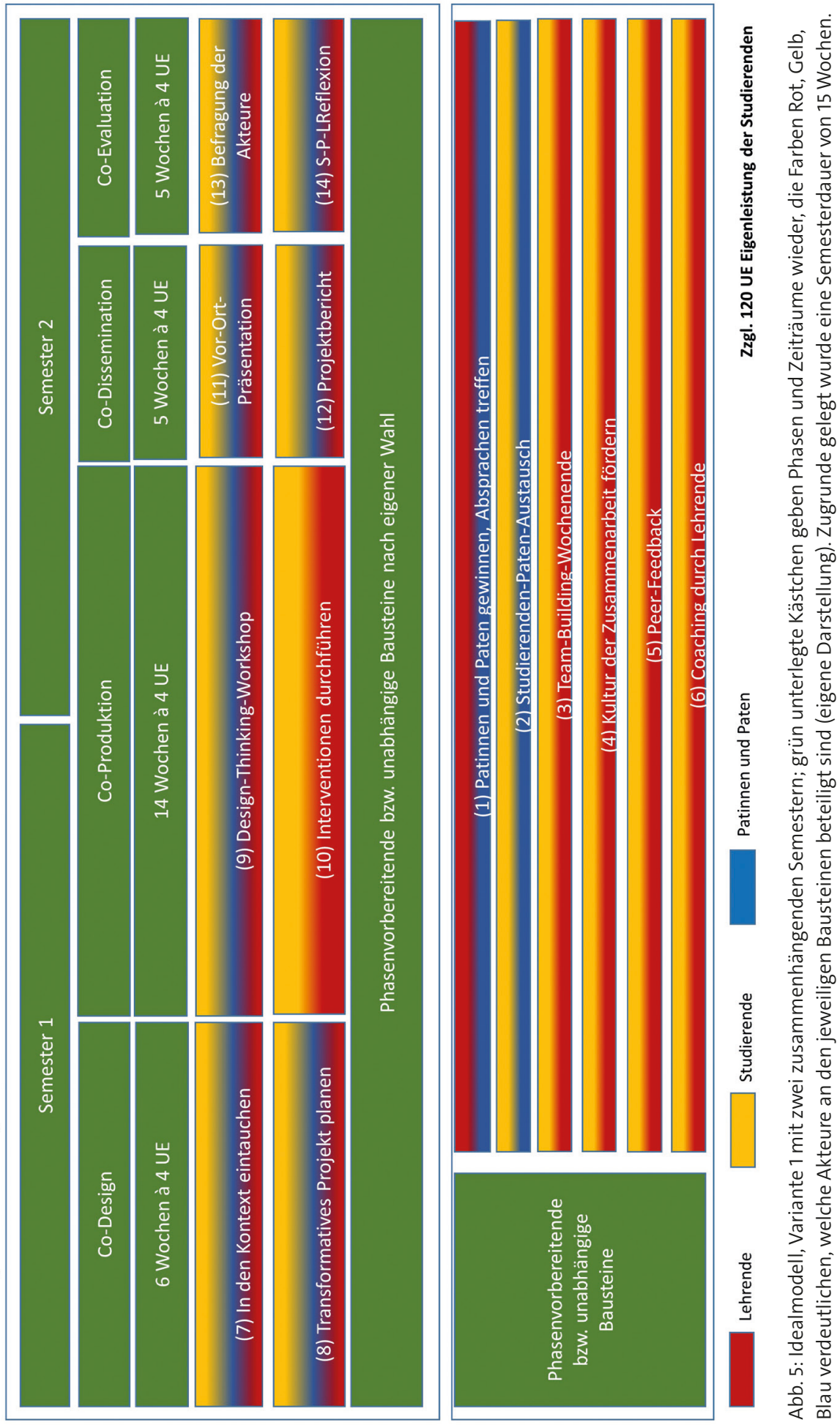


Nicht immer ist es möglich, ein zweisemestriges Modul mit einer konstanten Studierendengruppe zu planen (siehe Kap. 3). Zwei Alternativen sind in diesem Fall denkbar:

1) Alternative 1 (ohne eigene Abbildung, analog zu Abb. 5): Um allen Studierenden den Einblick in alle Phasen eines Projektes zu ermöglichen, empfiehlt es sich, die Inhalte in einem zeitlich kürzeren, dafür intensiveren Kurs innerhalb eines Semesters zu bearbeiten. Hier ist darauf zu achten, die Bausteine entsprechend schlank zu gestalten und die Anforderungen an die Studierenden dem geringeren Zeitumfang anzupassen.

2) Alternative 2 (siehe Abb. 6): Hier erfolgt eine "Staffelübergabe« zwischen zwei Studierendengruppen. Beide Semester beinhalten einen eigenen Kursabschluss mit Reflexion. Der Vorteil dieser Variante ist das höhere Zeitbudget für die einzelnen Bausteine. Auch ist es - so Studierende des ersten Semesterdurchgangs dafür zu gewinnen sind - möglich, die »Staffelübergabe« in Form eines Peer-Teachings durchzuführen, was Studierende beider Kurse unserer Erfahrung nach als motivierend erleben. Nachteilig ist jedoch, dass die Studierenden jeweils nur Ausschnitte eines transformativen Projektes erleben. Auch von den Patinnen und Paten wird es mitunter als zeitaufwendig erlebt, sich auf wechselnde Gruppen einzustellen und Kenntnisse mehrfach vermitteln zu müssen. 


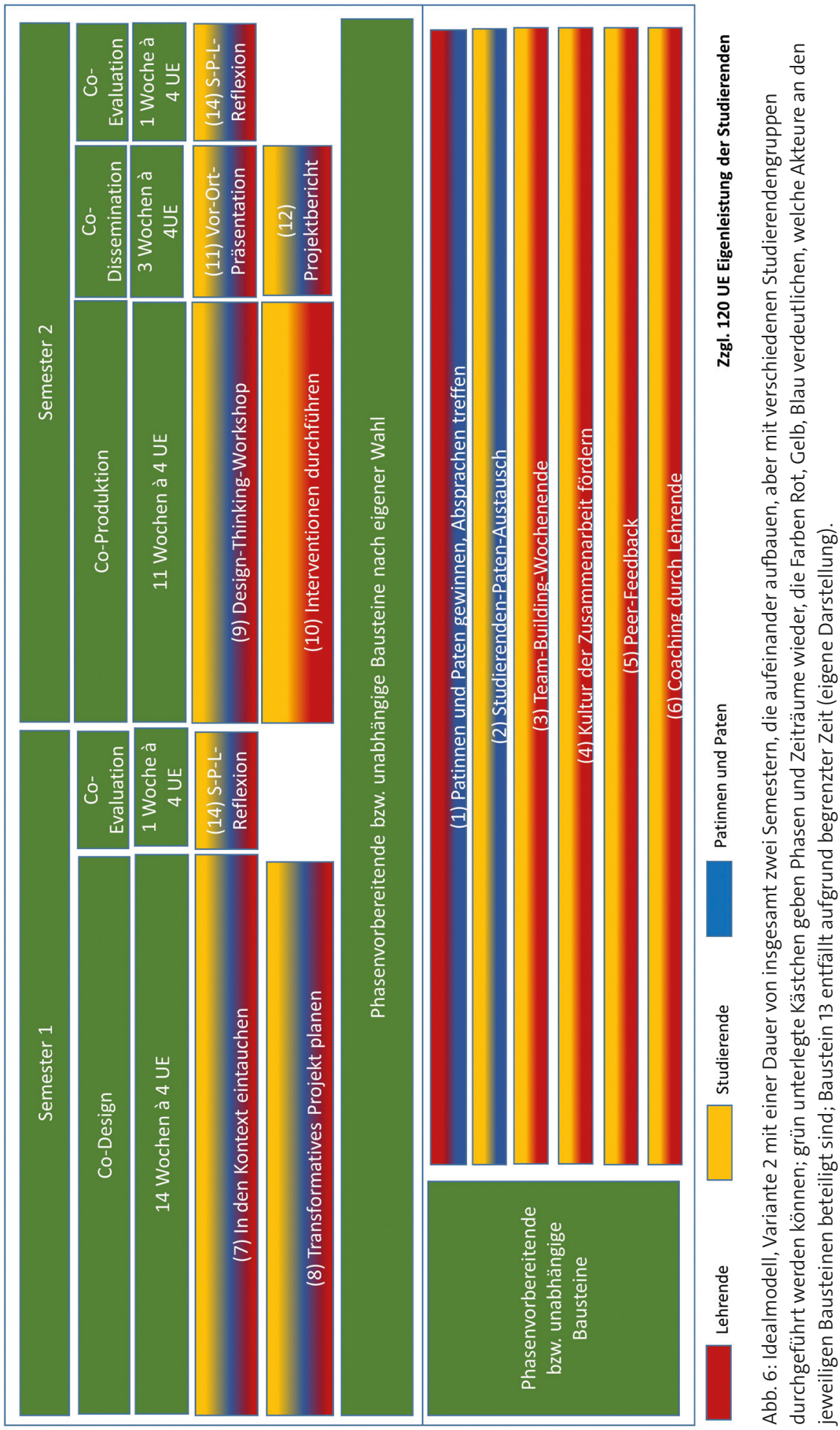




\section{Literaturverzeichnis}

Albiez M, König A, Potthast, T (2018) Transdisziplinarität und Bildung für Nachhaltige Entwicklung in der Lehre an der Universität Tübingen: Konzeptionelle Fragen mit Bezug auf Lehraktivitäten des »Energielabors Tübingen«. In: Leal Filho W (Hg.), Nachhaltigkeit in der Lehre: Eine Herausforderung für Hochschulen. Springer Spektrum, Berlin, S. 189-206 [https://doi.org/10.1007/978-3662-56386-1_12]

Beecroft R, Trenks H, Rhodius R, Benighaus C, Parodi O (2018) Reallabore als Rahmen transformativer und transdisziplinärer Forschung: Ziele und Designprinzipien. In: Defila R, Di Giulio A (Hg.) Transdisziplinär und transformativ forschen. Eine Methodensammlung. Springer VS, Wiesbaden: S. 75-99

Beecroft R (2020) Das Reallabor als transdisziplinärer Rahmen zur Unterstützung und Vernetzung von Lernzyklen. Dissertation, Leuphana Universität Lüneburg, Lüneburg

Berendt B, Voss HP, Wildt J (Hg.) Neues Handbuch Hochschullehre. Berlin, Stuttgart

Bergmann M, Jahn T (2005) Qualitätskriterien transdisziplinärer Forschung: Ein Leitfaden für die formative Evaluation von Forschungsprojekten. ISOE, Frankfurt a. M.

Bormann I, de Haan G (Hg.) (2008) Kompetenzen der Bildung für nachhaltige Entwicklung: Operationalisierung, Messung, Rahmenbedingungen, Befunde. VS Verlag für Sozialwissenschaften, Wiesbaden

BPB (Bundeszentrale für politische Bildung) (2011) Beutelsbacher Konsens. [https:// www.bpb.de/die-pb/51310/beutelsbacher-konsens] 
de Haan G (2008) Gestaltungskompetenz als Kompetenzkonzept der Bildung für nachhaltige Entwicklung. In: Bormann I, de Haan G (Hg.), Kompetenzen der Bildung für nachhaltige Entwicklung: Operationalisierung, Messung, Rahmenbedingungen, Befunde. VS Verlag für Sozialwissenschaften, Wiesbaden, S. 23-44

Defila R, Di Giulio A (2018) Reallabore als Quelle für die Methodik transdisziplinären und transformativen Forschens - eine Einführung. In: Di Giulio A, Defila R. (Hg.) (2018) Transdisziplinär und transformativ forschen. Eine Methodensammlung. Springer VS, Wiesbaden, S. 9-35

Ditton H, Müller A (Hg.) (2014) Feedback und Rückmeldungen: Theoretische Grundlagen, empirische Befunde, praktische Anwendungsfelder. Waxmann, Münster [u. a.]

Getzin S, Singer-Brodowski M (2016) Transformatives Lernen in einer Degrowth-Gesellschaft. Journal of Science-Society Interfaces 1(1): 33-46

GiBE und KommEnt (Gesellschaft für interkulturelle Bildungsforschung und Entwicklungspädagogik e. V. und KommEnt) (Hg.) (2016) Transformative Bildung angesichts der Post-2015-Debatte. Zeitschrift für internationale Bildungsforschung und Entwicklungspädagogik 39 (1)

Godat F, Müller-Geers M, Reisas S, Sandmann J (2018) Prototypen transdisziplinärer Lehrformate im Reallabor Kiel. HDS.Journal (1+2): 25-30

Grober U (2013) Die Entdeckung der Nachhaltigkeit. Kulturgeschichte eines Begriffs. Verlag Kunstmann, München

HRK und DUK (Hochschulrektorenkonferenz und Deutsche UNESCO-Kommission) (2010) Hochschulen für nachhaltige Entwicklung. Erklärung der Hochschulrektorenkonferenz (HRK) und der Deutschen UNESCO-Kommission (DUK) zur Hochschulbildung für nachhaltige Entwicklung. Ein Beitrag zur UN-Dekade »Bildung für nachhaltige Entwicklung« [https://www.hrk.de/fileadmin/redaktion/A4/ Hochschulen_und_Nachhaltigkeit_HRK_DUK.pdf]

Hilger A, Keil A (2021) Education for sustainable development with transdisciplinary-oriented courses - experiences and recommendations for future collaborations in higher education teaching. Journal of Geography in Higher Education [DOI: 10.1080/03098265.2021.1946765] 
Jürgens M, Golenia M (2020) E-Peer-Feedback zur Unterstützung Forschenden Lernens im Praxissemester - Konzept und Evaluationsergebnisse. In: Fischer B., Paul A. (Hg.) Lehren und Lernen mit und in digitalen Medien im Sport, CeBiS Schriftenreihe Vol. 18. Springer VS, Wiesbaden [http://doi-org-443.webvpn.fjmu. edu.cn/10.1007/978-3-658-25524-4_9]

Kropp A (2019) Grundlagen der Nachhaltigen Entwicklung. Handlungsmöglichkeiten und Strategien zur Umsetzung. Springer, Wiesbaden [https://link.springer.com/content/pdf/10.1007\%2F978-3-658-23072-2.pdf]

Krütli P, Pohl C, Stauffacher M (2018) Sustainability Learning Labs in Small Island Developing States: A Case Study of the Seychelles. GAIA - Ecological Perspectives for Science and Society 27(1): 46-51 [https://doi.org/10.14512/gaia.27.S1.11]

Lang DJ, Wiek A, Bergmann M, Stauffacher M, Martens P, Moll P, Swilling M, Thomas CJ (2012) Transdisciplinary research in sustainability science: Practice, principles, and challenges. Sustainability Science 7(S1): 25-43

Leal Filho W (2018) Identifizierung und Überwindung von Barrieren für die Umsetzung einer nachhaltigen Entwicklung an Universitäten: von Studienplänen bis zur Forschung. In: Leal Filho W (Hg.) Nachhaltigkeit in der Lehre: Eine Herausforderung für Hochschulen. Springer Spektrum, Berlin, S. 1-21

Meylan G, Lai A, Hensley J, Stauffacher M, Krütli P (2018) Solid waste management of small island developing states - the case of the Seychelles: a systemic and collaborative study of Swiss and Seychellois students to support policy. Environmental science and pollution research international 25(36):35791-35804 [https:// doi.org/10.1007/s11356-018-2139-3]

Müller-Christ G, Tegeler MK, Zimmermann CL (2018) Rollenkonflikte der Hochschullehrenden im Spannungsfeld zwischen Fach- und Orientierungswissen Führungstheoretische Überlegungen. In: Leal Filho W (Hg.) Nachhaltigkeit in der Lehre: Eine Herausforderung für Hochschulen. Springer Spektrum, Berlin, S. 51-68

Netzwerk n e.V. (2018) Methode n: Werkzeuge für eine nachhaltige Hochschule. [https://netzwerk-n.org/wp-content/uploads/2018/05/methode-n-2018-Digital-komprimiert.pdf] 
Pearce B, Adler C, Senn L, Krütli P, Stauffacher M, Pohl C (2018) Making the Link Between Transdisciplinary Learning and Research. In: Fam D, Neuhauser L, Gibbs P (Hg.) Transdisciplinary Theory, Practice and Education: The Art of Collaborative Research and Collective Thinking. Springer International Publishing, Cham, S. 167-184

Quartier Zukunft (Hg.) (2020): Dein Quartier und Du - Nachhaltigkeitsexperimente im Reallabor zu Nachbarschaften, Bienen, Naschbeeten, Kreativität und Konsum [DOI: 10.5445/KSP/1000076132]

RNM (Reallabor Nachhaltige Mobilität) (Hg.) (2018): Die Kultur des Experimentierens. In Reallaboren Nachhaltigkeit gemeinsam schaffen [http://www.r-n-m.net/ wp-content/uploads/2018/03/Forschen-mit-Realexperimenten.pdf]

Reis O (2009) Vom Reflex zur Reflexion - Prüfen und Bewerten von Prozessen reflexiven Lernens. In: Berendt B, Voss HP, Wildt J (Hg.) Neues Handbuch Hochschullehre. Berlin, Stuttgart: H 3.2

Rhodius R, Bachinger M, Koch B (Hg.) (2020) Wildnis, Wald, Mensch. Forschungsbeiträge zur Entwicklung einer Nationalparkregion am Beispiel des Schwarzwalds. oekom verlag, München [https://www.oekom.de/buch/wildnis-waldmensch-9783962380458]

Richter, A (2006): Portfolios im universitären Kontext: wann, wo, wie? Eine andere Bewertungsgrundlage im Seminarraum. In: Brunner I, Häcker T, Winter F (Hg.) Das Handbuch Portfolioarbeit: Konzepte, Anregungen, Erfahrungen aus Schule und Lehrerbildung, Kallmeyer bei Friedrich, Seelze-Velber, S. 234-241

Rose M, Wanner M, Hilger A (2019): Das Reallabor als Forschungsprozess und -infrastruktur für nachhaltige Entwicklung. Wuppertal Paper 196, Wuppertal

Schneidewind U, Singer-Brodowski M (2014) Transformative Wissenschaft - Klimawandel im deutschen Wissenschafts- und Hochschulsystem. 2., verb. und aktualisierte Aufl., Metropolis, Weimar

Singer-Brodowski M, Beecroft R, Parodi O (2018) Learning in Real-World Laboratories: A Systematic Impulse for Discussion. GAIA - Ecological Perspectives for Science and Society 27(1): 23-27 
Stöhler C, Förster C, Brehm L (2018) Projektmanagement lehren. Studentische Projekte erfolgreich konzipieren und durchführen [https://doi.org/10.1007/978-3658-18279-3]

Thiele K, Becker S (2020) Transformative Lehre für eine kritisch-geographische (Berufs-)Praxis. Standort 44: 268-274

Uebernickel F, Brenner W, Naef T, Pukall B, Schindlholzer B (2015) Design Thinking: Das Handbuch. Frankfurter Allgemeine Buch, Frankfurt

UN (United Nations) (2015) Transforming our world. The 2030 Agenda for Sustainable Development. A/RES/70/1, [https://sustainabledevelopment.un.org/ content/documents/21252030\%20Agenda\%20for\%20Sustainable\%20Development\%20web.pdf]

Wanner M, Schmitt M, Fischer N, Bernert P (2020) Transformative innovation lab: handbook to facilitate students' real-world laboratory projects to promote transformative and transdisciplinary competencies. Wuppertal Institut für Klima, Umwelt, Energie, Wuppertal [https://nbn-resolving.org/urn:nbn:de:bsz:wup4opus-76857]

WBGU (Wissenschaftlicher Beirat der Bundesregierung Globale Umweltveränderungen) (2011) Welt im Wandel: Gesellschaftsvertrag für eine Große Transformation. WBGU

West C (2018) »Wissen to Go« - Transdisziplinär-transformative Lehre als »Reallabor im Kleinen«. In: Di Giulio A, Defila R (Hg.) Transdisziplinär und transformativ forschen. Eine Methodensammlung. Springer VS, Wiesbaden, S. 329-373

Wiek A, Withycombe L, Redman CL (2011) Key competencies in sustainability: a reference framework for academic program development. Sustainability Science 6(2): 203-218

Zentrum für Hochschul- und Qualitätsentwicklung an der Universität DuisburgEssen (2014) Diversität konkret - Handreichung für das Lehren und Lernen an Hochschulen, Feedback-Methodenbar. [https://duepublico2.uni-due.de/ servlets/MCRFileNodeServlet/duepublico_derivate_00036794/07_Feedback_ Methodenbar.pdf] 


\section{Die Autorinnen}

Regina Rhodius, Umweltsozialwissenschaftlerin, forscht und lehrt im Umwelt- und Nachhaltigkeitskontext und betreut Projekte mit einem transformativ-transdisziplinären Ansatz. Ihre besondere Expertise liegt in der Gestaltung von Beteiligungsprozessen. Sie war an der Albert-Ludwigs-Universität Freiburg als Koordinatorin des Reallabors Wissensdialog Nordschwarzwald tätig.

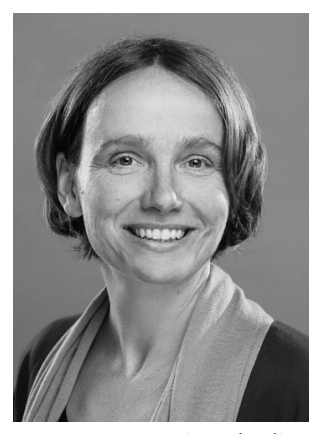

(c) Regina Rhodius

Monika Bachinger, promovierte Betriebswirtschaftlerin, ist als Professorin für Tourismus an der Hochschule für Forstwirtschaft in Rottenburg tätig. Ihre Forschungsschwerpunkte liegen in den Bereichen Tourismus in Großschutzgebieten, kulturelle Ökosystemleistungen, Destinationsmanagement, sowie Unternehmertum in kleinen und mittleren Unternehmen. Im Projekt Wissensdialog Nordschwarzwald war sie mit der Leitung der Teilprojekte „waldbasierter Gesundheitstourismus" und "Gründerinnen in Nationalparkregionen" betraut.

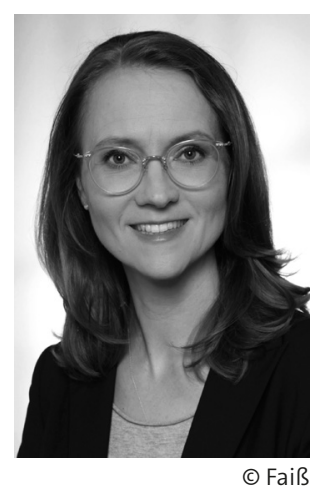



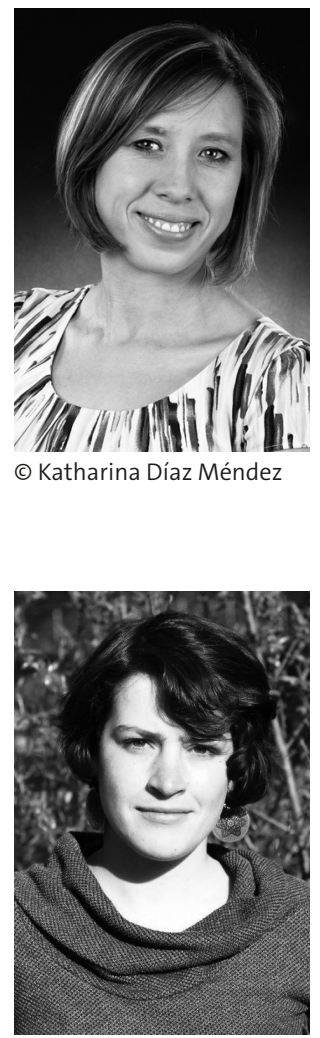

() Theresia Ziegler
Katharina Díaz Méndez, Geistes- und Sozialwissenschaftlerin, arbeitet seit 2017 an der Schnittstelle von Wissenschaft und Praxis an der Hochschule für Forstwirtschaft Rottenburg mit Schwerpunkt BNE und Entrepreneurship. Im Reallabor Wissensdialog Nordschwarzwald war sie als wissenschaftliche Mitarbeiterin in den Teilprojekten „waldbasierter Gesundheitstourismus" und „Gründerinnen in Nationalparkregionen“ tätig.

Susanne Ober studiert im Master Nachhaltigkeitswissenschaften an der Leuphana Universität Lüneburg und forscht im Rahmen der Masterarbeit zu transdisziplinären Nachhaltigkeitsprojekten. Im Reallabor Wissensdialog Nordschwarzwald nahm sie zunächst als Studierende teil und war später als Teil des Reallaborteams selbst als Lehrende aktiv. 